\title{
Equidad en la Accesibilidad Geográfica a los Hospitales. El Caso del País Vasco
}

\section{Equity in the Geographical Accessibility to Hospitals. The Case of de Basque Country}

\author{
Javier Cano-Fernández \\ javier.canofernandez@osakidetza.eus @ 0000-0003-4414-4017 \\ Osakidetza, Red de Salud Mental de Bizkaia, Área Económico-Financiera. \\ C/ María Díaz de Haro, 5848010 Bilbao (Bizkaia). \\ Marisol Esteban-Galarza \\ marisol.esteban@ehu.eus @ 0000-0002-4553-1126 \\ Universidad del País Vasco / Euskal Herriko Universitatea, Facultad de Economía y Empresa, \\ Departamento de Políticas Públicas e Historia Económica. \\ Avda. Lehendakari Agirre, 83. 48015 Bilbao (Bizkaia).
}

\section{INFO ARTÍCULO}

Recibido: 23-11-2020

Revisado: 17-01-2021

Aceptado: 19-01-2021

\section{PALABRAS CLAVE}

\section{Equidad geográfica}

Eficiencia

Salud

País vasco

Osakidetza

\section{KEYWORDS}

Geographical equity

Efficiency

Health

Basque country

Osakidetza

\begin{abstract}
RESUMEN
El objetivo del presente artículo es analizar la equidad espacial y la eficiencia de la distribución geográfica de los recursos hospitalarios del País Vasco gestionados por el Servicio Vasco de Salud (Osakidetza), bajo el prisma del nuevo modelo de organización sanitaria integrada. Usando Google Earth para la localización espacial y extracción de coordenadas geográficas, se realiza un análisis estadístico de accesibilidad poblacional, considerando tiempo y distancia hasta el recurso de Atención Especializada referente. A la vista de los resultados ha sido posible evidenciar la existencia, en general, de un alto nivel de equilibrio entre la equidad espacial y la eficiencia citados, puesto que el $90 \%$ de la población dispone de una accesibilidad temporal y espacial calificable entre óptima y normal, con un tiempo de acceso de 0 a 20 minutos o una distancia entre 0 y $20 \mathrm{~km}$. Por tanto, teniendo en cuenta lo expuesto, las acciones de mejora deberán orientarse hacia el $10 \%$ de la población más desfavorecida, con iniciativas como la redefinición del mapa sanitario; establecimiento de convenios de colaboración con otras regiones; mejora de las infraestructuras de comunicaciones, sin excluir otras acciones que puedan alcanzar el mismo objetivo.
\end{abstract}

\begin{abstract}
The aim of this article is to analyse the spatial equity and efficiency of the geographical distribution of the hospital resources of Basque Country manage by the Basque Health Service (Osakidetza), under the prism of the new model of Integrated Healthcare Organisation. Using Google Earth for spatial location and extraction of geographical coordinates, a statistical analysis of population accessibility is carried out, considering time and distance to the reference Specialised Care resource. In view of the results, it has been possible to demonstrate the existence, in general, of a high level of balance between the spatial equity and efficiency mentioned, since $90 \%$ of the population has a temporal and spatial accessibility that can be qualified as between optimum and normal, with an access time of 0 to 20 minutes or a distance of between 0 and $20 \mathrm{~km}$. Therefore, taking into account the above, the improvement actions should be directed towards the $10 \%$ of the most disadvantaged population, with initiatives such as the redefinition of the health map; the establishment of collaboration agreements with other regions; the improvement of communication infrastructures, without excluding other actions that could achieve the same objective.
\end{abstract}




\section{INTRODUCCIÓN}

Etimológicamente la palabra "equidad" procede del término latino "æquitas"-atis" que pudiera traducirse por "igualdad"; pero es la tercera acepción del diccionario de la RAE: "justicia natural, por oposición a la letra de la ley positiva", la que creemos se ajusta a nuestro planteamiento.

En el ámbito de la salud, desde la Organización Mundial de Salud, se enuncian algunos conceptos y principios de equidad, referidos a que todas las personas deben contar con similares oportunidades para alcanzar todo su potencial en salud. Por tanto, se trata de una cuestión de medios, más que de resultados, ya que no se pretende conseguir, en todo caso, el mismo nivel y calidad en cuanto a salud, sino la eliminación de aquellas diferencias injustas que puedan ser evitables, dotando a las personas de las mismas oportunidades para alcanzar la salud (Whitehead, 1991).

Por ello, la equidad es un referente ético y como tal debe considerarse en las decisiones que se adopten referidas a la financiación y en el destino del gasto sanitario, en particular, como a cualquier política pública, en general, y en el ámbito de la salud puede interpretarse desde una perspectiva poliédrica, atendiendo a los diferentes aspectos que puede contemplar. A este respecto, sin pretender ser exhaustivos, pueden enumerarse, a modo de ejemplo: equidad de género, en función de las necesidades específicas de cada sexo; según Almeida, (2013) la equidad horizontal "es la atención igual para las personas con igual necesidad, independientemente de las características tales como ingreso, clase social, años de educación, raza, etnia, lugar de residencia, entre otros factores socio-económicos", y al contrario que la anterior, según Barbieri, Puente y Tarragona, (2002), equidad vertical es la que "establece que situaciones diferentes merecen ser tratadas de forma diferentes"; equidad en la prestación sanitaria, es la referida a los recursos disponibles (medios empleados), en contraposición a la equidad en salud, que compara los resultados conseguidos.

La contrapartida y también derivada de la equidad, es la eficiencia, la cual mide la relación existente entre los resultados obtenidos y los recursos consumidos en la ejecución de un proyecto dado; maximizar la eficiencia consiste en hacer más con menos, sin importar en principio, quien resulta beneficiado o perjudicado con la decisión sobre el destino de los recursos. Por tanto, en aras de la sostenibilidad económica de los sistemas sanitarios, es necesario priorizar el gasto, sopesando ambos principios: equidad y eficiencia, pero sin descuidar el coste-efectividad en la aplicación de los recursos sanitarios.

En el caso que nos ocupa, el efecto o resultado que supone para la población encontrarse con una determinada accesibilidad temporal o territorial (tiempo o distancia), es la medida de la eficiencia. De tal forma que una organización con recursos sanitarios equivalentes a otras, que proporcione tiempos o distancias mejores hasta su hospital de referencia y a un número mayor de usuarios, será más eficiente desde el punto de vista de la accesibilidad, pero quizá, no sea la más equitativa. Por ello, el punto de equilibrio entre ambos principios (eficiencia y equidad) el cual se objetivará matemáticamente, será el resultado del análisis multivariante que relacione en una ecuación, el tiempo y distancia citados, con la población municipal. El coeficiente de variación será la medida de la equidad, realizándose el análisis a todos los niveles (Comunidad Autónoma, Provincia y organización sanitaria integrada de referencia).

Desde su vertiente geográfica, el concepto de equidad puede explicarse por contraste con su antónimo; únicamente en el supuesto que una comarca o municipio, con respecto a otras, se encuentre más distante de los recursos sanitarios en un grado mayor, de lo que el tamaño de su población nos permita esperar razonablemente, pudiéramos encontrarnos ante un supuesto de inequidad geográfica en el acceso a los recursos sanitarios; todo ello sin perder de vista que los recursos económicos disponibles son escasos y las necesidades, igual que la demanda de prestaciones sanitarias, ilimitadas. No obstante, las soluciones no corresponden en exclusiva a la Administración Sanitaria, sino que, a veces, pasan por la mejora de las vías de comunicación o servicios de transporte público.

Así que no debe considerarse, en cualquier caso, una desigualdad injusta el hecho que los recursos sanitarios no alcancen a las áreas rurales, menos habitadas, en la misma medida que a otras zonas con mayor población; pero tampoco se debe perder de vista la relación entre accesibilidad y equidad en la cohesión

1. Real Academia Española. 
territorial, tal y como Camarero y Oliva, (2019) evidencian: los mapas de prestación de servicios, redes de infraestructuras e inversiones, no sólo remodelan territorios sino también sus morfologías sociológicas.

Visto lo cual, el objetivo de este artículo, y pensamos también, su principal aportación y justificación, es analizar si la distribución geográfica de los hospitales en el País Vasco, dependientes del Servicio Vasco de Salud (Osakidetza), se encuentran repartidos adecuadamente desde el punto de vista de la equidad espacial y la eficiencia y, además, si son compatibles con las necesidades hospitalarias de la población bajo el prisma organizativo del modelo de organización sanitaria integrada, permitiendo el análisis comparado entre las distintas organizaciones sanitarias integradas.

El alcance del artículo, por tanto, se referirá a la accesibilidad geográfica, medida en función del tiempo y la distancia que precisa un ciudadano para acudir, desde el centro de Atención Primaria más cercano a su domicilio, hasta el hospital correspondiente a la organización sanitaria integrada que tiene asignada según su lugar de residencia. Todo lo cual, por comparación entre organizaciones sanitarias integradas, también nos permitirá medir la eficiencia en el uso y distribución espacial de los hospitales del Servicio Vasco de Salud (Osakidetza), así como la equidad en la asignación geográfica de dichos recursos, contextualizando el marco territorial y población del País Vasco con su mapa sanitario.

La metodología de análisis y extracción de datos se ha llevado a cabo mediante el uso de la información obtenida de la aplicación Google Earth, elaborándose posteriormente con esos datos, un análisis estadístico descriptivo (media aritmética, desviación típica y coeficiente de variación) y multivariante (regresión simple), así como otro de accesibilidad poblacional, considerando el tiempo o distancia necesarios que precisa un ciudadano para acudir, desde su entorno sanitario próximo, al dispositivo de referencia de Atención Especializada correspondiente. Asimismo, el análisis multivariante se ha encuadrado con el tamaño de la población municipal.

Vistos los resultados obtenidos y el ulterior análisis de estos, la discusión versará sobre si se ha podido evidenciar, en el ámbito del estudio, la existencia de un razonable equilibrio entre eficiencia y equidad geográficas y como punto final, si procede, en las conclusiones llegar a plantear áreas de mejora para aquellas ubicaciones donde no se alcanza el resultado esperado.

El artículo se estructura en seis secciones, al margen de bibliografía, las cuales son: 1. Introducción; 2. Marco teórico y estado de la cuestión; 3. País Vasco. Marco territorial de estudio y organización sanitaria, dividido a su vez en las siguientes subsecciones: 3.1. Marco territorial del estudio: País Vasco, 3.2. Modelo de organización sanitaria integrada, 3.3. Mapa sanitario de Osakidetza; 4. Metodología; 5. Resultados y discusión; 6. Conclusiones.

\section{MARCO TEÓRICO Y ESTADO DE LA CUESTIÓN}

La ubicación física de los equipamientos públicos y servicios comunitarios, especialmente los sanitarios, educativos y sociales, en el momento de la decisión de su localización, debe contemplar un compromiso equilibrado entre equidad y eficiencia, maximizando ambos parámetros simultáneamente, sin que la mejora del uno, al mismo tiempo suponga un menoscabo inaceptable del otro. A este respecto, el concepto de justicia territorial o espacial en la decisión de la localización de los equipamientos públicos ha sido materia de trabajo en tesis, artículos científicos etc., haciendo converger la ciencia geográfica con la distribución de los recursos sanitarios y junto a ellos, en ocasiones, también la sostenibilidad económica. Así pues, ya existe literatura precedente que aúna los conceptos equidad espacial y eficiencia, la cual también será la línea de investigación que desarrollará este trabajo, aunque en este caso con una metodología diferente, circunscrita a la Comunidad Autónoma del País Vasco.

Entre muchos otros trabajos, resulta relevante en el caso del sistema sanitario español revisar las aportaciones de Basoa y Otero (1994), quienes en su momento, elaboraron un novedoso estudio sobre la "Accesibilidad geográfica a los centros de salud y planeamiento urbanístico en Fuenlabrada (Madrid)" permitiendo conocer el nivel de accesibilidad al Área de Salud, evidenciándose la necesidad de planificar, a futuro, "Ios equipamientos sanitarios en función de las determinaciones y previsiones de los planes urbanísticos". De 
temática afín, aunque referido a la Comunidad Autónoma de Andalucía, López y Garrido, (2003), elaboraron un estudio sobre la accesibilidad hospitalaria, hasta los hospitales de dicha comunidad, mediante la medición, por carretera, de la distancia espacial y temporal.

Más recientemente, también es destacable, el estudio de López et al., (2012) quienes realizaron un análisis, utilizando un Sistema de Información Geográfica y como resultado una propuesta de accesibilidad y ubicación territorial óptima de los centros sanitarios, en el ámbito de la salud mental de Andalucía. En esa misma Comunidad Autónoma, la Tesis Doctoral de Rodríguez (2014) sobre "Accesibilidad geográfica de la población a la red de hospitales públicos de Andalucía: aportaciones desde el análisis de redes", destaca la importancia de la aplicación de la accesibilidad geográfica a los recursos asistenciales especializados, tanto para su ubicación como para ordenar los servicios sanitarios.

Al margen del caso español, debe ser reseñada la aportación de Loyola et al., (2002) con relación a “los Sistemas de Información Geográfica como herramientas para monitorear las desigualdades en salud"y como instrumento de planificación de la política sanitaria, al igual que el artículo de Gang et al., (2016), quienes analizan la accesibilidad espacial de cada subdistrito de la ciudad de Shenzhen (China) hasta sus hospitales primarios, secundarios y terciarios, en función del tiempo de viaje y diferencia espacial, tanto en transporte público como vehículo privado. Asimismo, en ese país, Pan, Zhen y Jing, (2019) evalúan la equidad y la eficiencia de la distribución espacial de los servicios básicos de salud en el área metropolitana de Nanjing (China).

Por otro lado, la equidad geográfica, también ha tenido múltiples enfoques y así Whitehead et al., (2019), en su artículo basado en un análisis bibliográfico sobre la materia, no han encontrado un consenso claro sobre las definiciones o medidas de equidad espacial de los servicios de salud, cuando analizaron setenta y cinco estudios relacionados con la materia, en la mayoría de los casos la medición de la equidad espacial se llevó a cabo aplicando el coeficiente de Gini en conjunción con un análisis de correlación espacial.

Hay otros autores que plantean la equidad en salud desde la visión de las desigualdades territoriales englobadas en una suerte de geografía de la salud, "como disciplina que estudia la distribución territorial de la salud" (Tobar et al., 2001).

Por tanto, para que el objetivo de que la distribución de infraestructuras sanitarias sea eficiente a la par que equitativa, es necesario cumplir lo que en el ámbito económico y de las Ciencias Sociales se ha denominado como el Óptimo de Pareto, por el que se suscita una encrucijada entre eficiencia y equidad, solventando el óptimo individual, pero no el óptimo social, ya que además de la asignación de recursos, es importante que estos se encuentren distribuidos equitativamente. (Reyes y Franklin, 2016).

Por eso, el punto de equilibrio debiera ser el acercamiento temporal y en su caso espacial, de los recursos asistenciales beneficiando a un mayor número de ciudadanos, sin perjuicio del mantenimiento de un umbral mínimo de equidad que proporcione una dotación de medios y accesibilidad suficiente a los que por dispersión geográfica, aislamiento o despoblamiento, no dispongan de una alternativa más próxima, tal como lo expresa Betancourth, (2011), aunque no es posible para toda la ciudadanía, tener al mismo tiempo idéntico nivel de accesibilidad sanitaria, ya que los lugares de residencia de unos y otros tienen diferente densidad de población, la equidad se puede considerar óptima, cuando se maximiza el mayor número de ciudadanos con acceso suficiente a los servicios sanitarios.

En el mismo sentido que el anterior, apuntan Ramírez y Bosque, (2001), citando a su vez a Smith, (1980), quienes ponen de manifiesto que la eficiencia y la equidad pueden, en la práctica, ser contrapuestos ya que la eficiencia óptima puede dar lugar a un resultado distributivo no equitativo. De todo ello es posible concluir, que casi seguro resulta imposible, ubicar una infraestructura pública, beneficiando igualmente a toda la ciudadanía. Por todo ello las decisiones sobre la ubicación deben ser sopesadas cuidadosamente, teniendo en cuenta las necesidades y distribuyendo las cargas y beneficios lo más equitativamente posible. 


\section{PAÍS VASCO. MARCO TERRITORIAL DE ESTUDIO Y ORGANIZACIÓN SANITARIA}

\subsection{Marco territorial del estudio: País Vasco}

El País Vasco o Euskadi es una de las diecisiete comunidades autónomas en las que se organiza territorialmente el Estado Español, que a su vez está conformado por tres provincias o Territorios Históricos cuya denominación oficial, tanto en castellano como en lengua vasca (euskera) son: Araba/Álava, Bizkaia y Gipuzkoa3.

La localización se encuentra al norte de la Península Ibérica, en el extremo más oriental del Golfo de Vizcaya, a orillas del Mar Cantábrico. La superficie es de 7234,9 km², con una población total de 2175819 habitantes (2017) y una densidad de 300,7 hab/ $\mathrm{km}^{2}$ repartiéndose administrativamente entre 251 municipios, que a nivel de Territorio Histórico se resume4:

- Araba/Álava: 3041,8 km²/ 323592 hab. / 106,4 hab/km² / 51 municipios.

- Bizkaia: 2214,9 km²/1 139426 hab. / 514,4 hab / $\mathrm{km}^{2} / 112$ municipios.

- Gipuzkoa: 1978,3 km²/ 712801 hab. / 360,3 hab/km². / 88 municipios.

Es evidente que existe, entre los tres territorios, gran disparidad en cuanto a extensión territorial, población y densidad, acentuada aún más a nivel municipal y comarcal, con gradientes máximos entre las comarcas del Área Metropolitana de Bilbao (Gran Bilbao) con 857016 hab. / 2281,8 hab/km² y la Montaña Alavesa con 3009 hab. / 5,6 hab/km².

Estas circunstancias condicionan el Mapa Sanitario así como el modelo organizativo de Osakidetza que luego veremos, ya que la geografía del País Vasco, es reducida en extensión y la altitud media de la Comunidad, si bien no es muy elevada (232 msnm) ${ }^{5}$, la orografía es muy abrupta y montañosa, especialmente la de Gipuzkoa y en menor medida Bizkaia, lo cual influye en el trazado de las vías de comunicación que afectan a los desplazamientos de la población.

Pese a todo, el País Vasco cuenta con unas más que aceptables comunicaciones por carretera, con vías de gran capacidad, como la Autovía/Autopista del Cantábrico (A-8/AP-8) que une Bizkaia con Gipuzkoa; la Autopista Vasco-Aragonesa (AP-68) entre Bizkaia y Araba/Álava y la Autopista del Norte AP-1, entre Araba/Álava y Gipuzkoa. Asimismo, existen otras autovías, corredores supracomarcales y carreteras de orden menor que permiten unos desplazamientos fluidos.

Aun así, existen carencias en algunas comarcas cuyas carreteras no están a la altura del resto y en concreto y sin pretensión de una descripción exhaustiva, se puede decir que la situación más precaria está en la zona más occidental de la comarca vizcaína de las Encartaciones/Enkarterri y específicamente los municipios próximos a la Comunidad Autónoma de Cantabria, a los que no alcanza la autovía denominada corredor del Cadagua. En la misma situación se encuentra la comarca de Lea-Artibai (Markina-Ondarroa) en Bizkaia, la Montaña y Valles Alaveses, así como algunos municipios del interior de Gipuzkoa, los cuales también precisan una mejora de sus infraestructuras permitiendo tiempos de desplazamiento inferiores, al menos, hasta los núcleos de población que centralizan los servicios públicos.

2. Las denominaciones "Euskadi" " “País Vasco" son equivalentes y se refieren al mismo territorio que constituye la Comunidad Autónoma (artículo 1 de la Ley Orgánica 3/1979, de 18 de diciembre de Estatuto de Autonomía del País Vasco). Publicada en el BOE núm. 306, de fecha 22 de diciembre de 1979: https://www.boe.es/buscar/pdf/1979/BOE-A-1979-30177-consolidado.pdf

3. Ley 19/2011, de 5 de julio, por la que pasan a denominarse oficialmente [también en castellano] "Araba/Álava”, "Gipuzkoa” y “Bizkaia" las demarcaciones provinciales Ilamadas anteriormente "Álava", "Guipuzcoa” y “Vizcaya”. Publicada en el BOE núm. 160, de fecha 06/07/2011: https://www.boe.es/boe/dias/2011/07/06/pdfs/BOE-A-2011-11606.pdf

4. Instituto Vasco de Estadística (Eustat), 2017.

5. Estadística del País Vasco: http://es.db-city.com/Espa\%C3\%B1a--Pa\%C3\%ADs-Vasco 


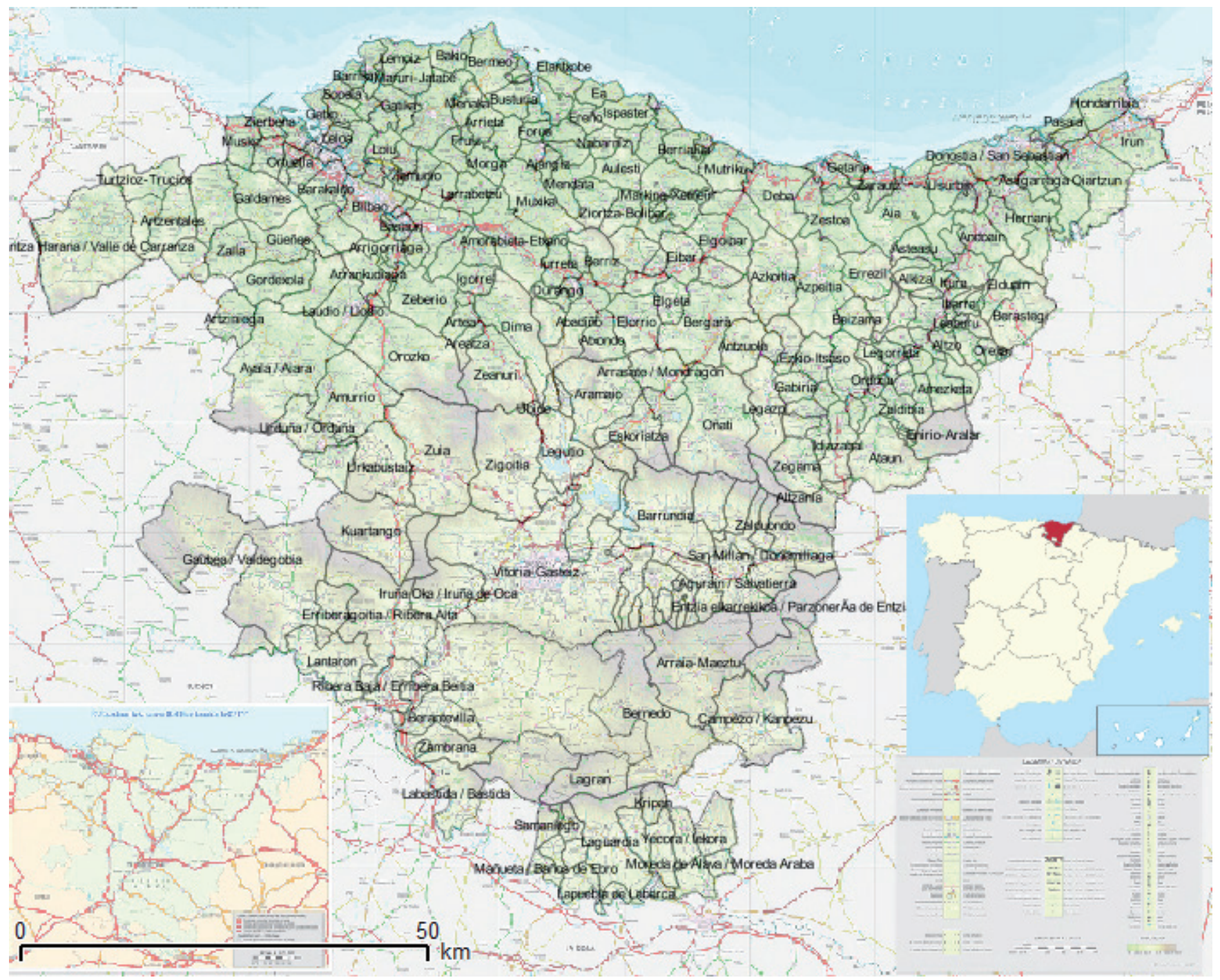

Mapa 1. Mapa General del País Vasco. Fuente: Elaboración propia (combinando y superponiendo el Mapa de Carreteras 2014 y Mapa Vectorial de Límites Administrativos CAPV, obtenidos de Gobierno Vasco (Eusko Jaurlaritza). geoEuskadi con el Mapa de España miniaturizado, obtenido de (Wikipedia).

\subsection{Modelo de organización sanitaria integrada}

En 1983 se crea en el ámbito público, el Servicio Vasco de Salud (Osakidetza), que desde entonces se ha encargado de la provisión de las prestaciones sanitarias públicas en el ámbito de la CAPV. Posteriormente, en el periodo que va del año 2011 hasta el 2016, es cuando se concluye el completo despliegue de un nuevo modelo organizativo y se emprende una reforma de la estructura organizacional, evolucionando hacia el nuevo paradigma asistencial de organización sanitaria integrada. Tanto en la exposición de motivos del Acuerdo del Consejo de Administración de Osakidetza por el que se creaba la primera de estas, la organización sanitaria integrada Bidasoa, como en la nota de prensa del Gobierno Vasco en su canal de comunicación Irekia de 30/01/2014 "Osakidetza crea tres nuevas [organizaciones sanitarias integradas] en Bizkaia" se justificaban las razones y objetivos del nuevo modelo:

- Se supera la fragmentación entre Atención Especializada y Primaria, integrando en una sola organización los Hospitales y la Comarca de Atención Primaria de su demarcación geográfica.

- Facilita la colaboración entre profesionales y servicios asistenciales, trabajo en equipo, compartir protocolos de actuación e información clínica, todo ello bajo una dirección única y conjunta. 
- Es una alternativa a los problemas de ineficiencia y duplicidades en la gestión de recursos y la pérdida de continuidad en los procesos asistenciales, ofreciendo una mejor atención al paciente, quien no percibe fisuras entre ambos niveles sino una continuidad y coherencia en su cuidado.

- Mayor orientación al paciente, permitiendo afrontar el reto de la cronicidad y el progresivo envejecimiento de la población, frente al abordaje tradicional del paciente agudo.

\subsection{Mapa sanitario de Osakidetza}

El número de organizaciones sanitarias integradas es trece, de acuerdo con la siguiente distribución (dos en Araba/Álava, cinco en Bizkaia y seis en Gipuzkoa) las cuales, en total, incluyen 13 hospitales de agudos, 153 centros de salud y 160 consultorios $^{6}$, así como otros dispositivos sanitarios:

Tabla 1. Organizaciones sanitarias integradas por Área de Salud.

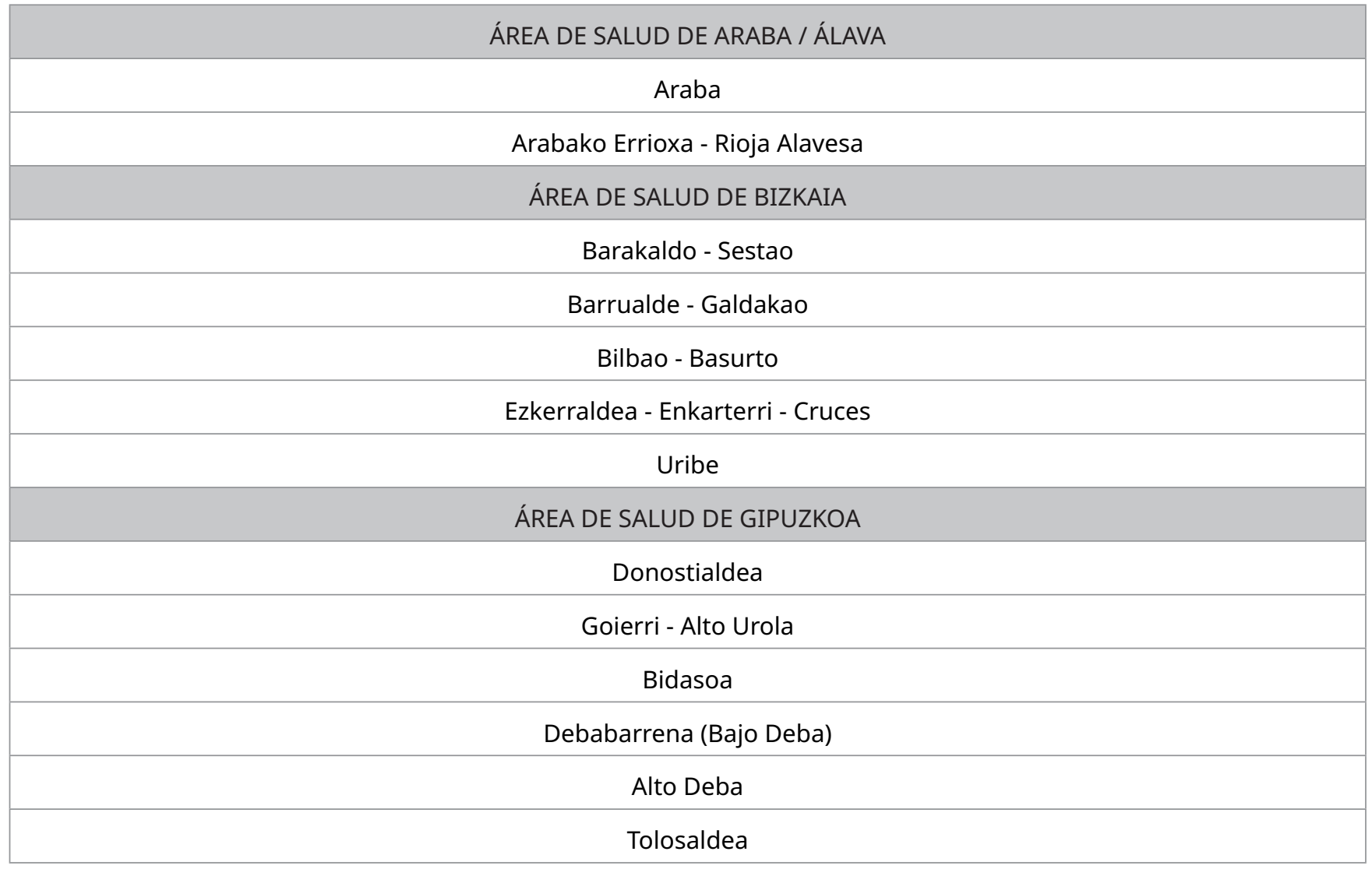

Fuente: Elaboración propia, a partir de datos de Osakidetza.

Al margen de su denominación oficial, lo cierto es, que la principal diferencia entre centros de salud y consultorios, se debe a que los primeros se ubican en los distritos de las capitales provinciales y municipios de mayor población. Su cartera de servicios asistencial, generalmente más amplia, incluye en algunos casos Atención Especializada ambulatoria para ciertas patologías y el horario de atención al público también es superior. Por el contrario, los consultorios se encuentran en zonas rurales, con horario de atención al público, inferior y adaptados a la reducida población que tienen que atender. Su cartera de servicios también es más escasa y se limitan, generalmente, a la Atención Primaria (medicina familiar, enfermería y otras prestaciones concurrentes).

Cada una de las organizaciones sanitarias integradas cuenta, al menos, con un hospital general de agudos para las prestaciones de Atención Especializada hospitalaria y para la Atención Primaria, un número de

6. Recursos del Sistema Sanitario Público Vasco: https://www.etxebide.euskadi.eus/x39-contgen/es/contenidos/informacion/recursos_sistema_sanitario/es_sanidad/index.shtml 
centros de salud y consultorios acordes a su población y extensión territorial. Los hospitales del Servicio Vasco de Salud (Osakidetza) son:

- Araba/Álava: 2 hospitales generales de agudos, en su capital, Vitoria, y otro hospital más en Leza, así como 1 hospital de salud mental, también en Vitoria.

- Bizkaia: 5 hospitales generales de agudos (1 Bilbao, 2 Barakaldo, 1 Galdakao y 1 Urduliz). Además, esta provincia cuenta con un hospital de alta resolución en Gernika, otros dos de crónicos y cuidados paliativos en Bilbao y Gorliz y 3 hospitales de salud mental en Bermeo, Zaldibar y Zamudio respectivamente.

- Gipuzkoa: 6 hospitales generales de agudos (1 Donostia/San Sebastián, 1 Mendaro, 1 Mondragón, 1 Zumárraga, 1 Tolosa y 1 Hondarrabía/Irún) y 1 hospital de crónicos en Eibar.

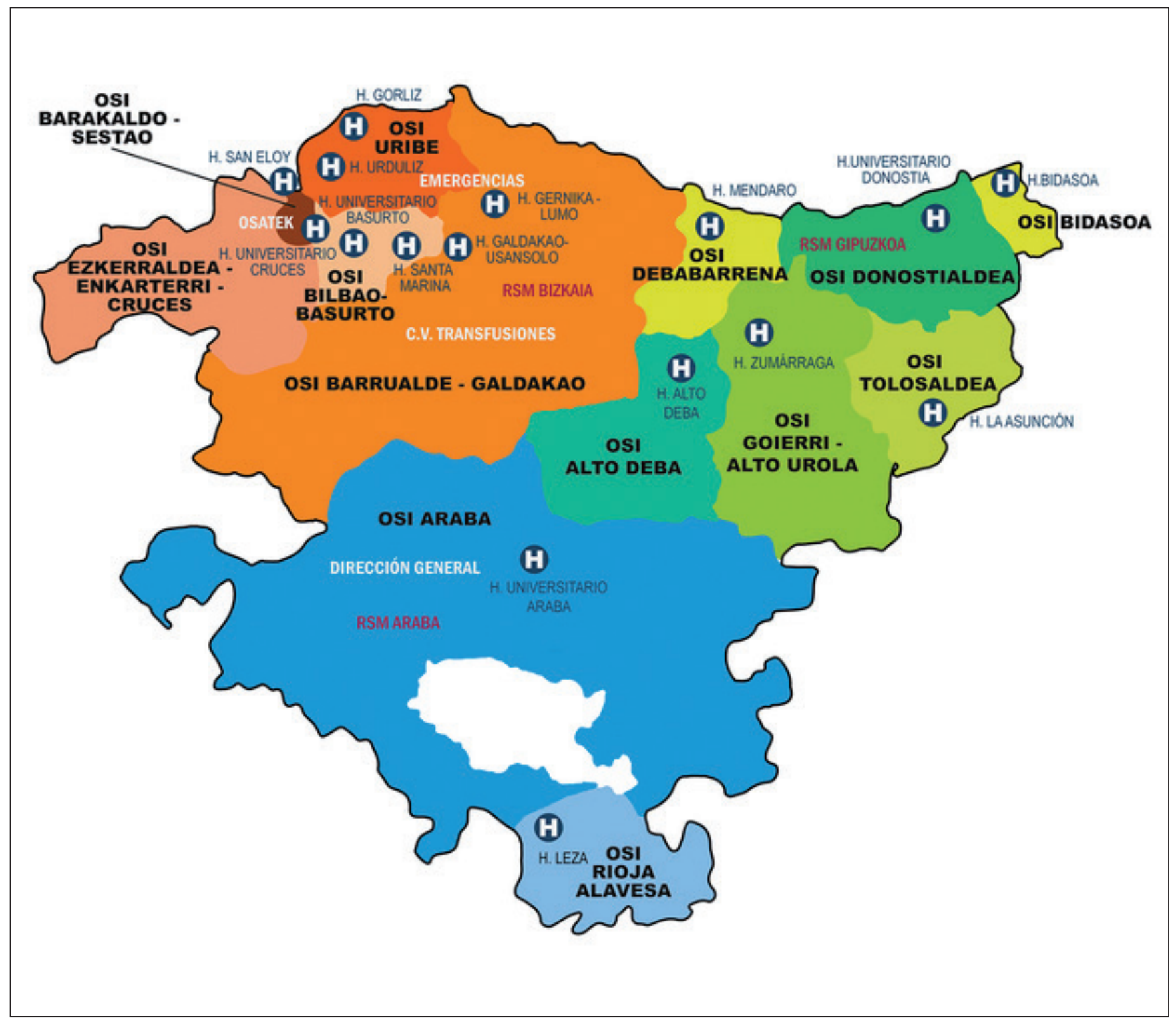

Mapa 2. Mapa de organizaciones sanitarias integradas y hospitales. Fuente: Obtenido de "Recursos del Sistema Sanitario Público Vasco”. 


\section{METODOLOGÍA}

Para alcanzar el objetivo propuesto, en primer lugar, se ha obtenido la dirección exacta y completa de todos y cada uno de los centros de salud, consultorios y hospitales, disponible en el directorio de centros de Osakidetza ${ }^{7}$. A continuación, se procede a su ubicación en el software geográfico Google Earth verificando cada caso mediante la opción Street View y cotejando que las imágenes del dispositivo sanitario registradas en la aplicación, se correspondan con la dirección del directorio. En una siguiente fase, se obtienen las coordenadas geográficas y se registra el tiempo y la distancia hasta el hospital correspondiente, mediante la extracción de los archivos de localización referenciados geográficamente "Keyhole Markup Language" (KML) y su formato comprimido (KMZ). La aplicación Google Earth permite extraer las coordenadas, sin necesidad de otras herramientas informáticas más complejas. Además, puede conseguirse un resultado de medición óptimo de los parámetros tiempo y distancia, sobre el mapa virtual de la red de carreteras del programa.

Posteriormente, estos archivos son exportados al Sistema de Información Geográfica QGIS, para su tratamiento y realización de los mapas de elaboración propia incluidos en este artículo.

A diferencia de otros trabajos sobre localización espacial óptima de infraestructuras comunitarias, que por regla general utilizan "centroides de radios censales como puntos de demanda y sitios candidatos para la localización de la oferta de servicios", (Buzai, 2011), en nuestro caso hemos asumido que la ubicación de los equipamientos de Atención Primaria existentes, ya se encuentran distribuidos territorialmente de manera homogénea a lo largo y ancho del territorio del País Vasco. Estos constituyen los recursos más próximos al domicilio de los usuarios, por lo que resulta posible utilizar su localización como patrón o punto de partida para la comparación temporal y espacial hasta su hospital de referencia de la organización sanitaria integrada.

La metodología aplicada es, en primer lugar, la realización de un análisis estadístico descriptivo y más tarde multivariante, considerando el tiempo y la distancia más breve, necesarios para desplazarse desde el dispositivo asistencial de Atención Primaria (centro de salud/ambulatorio o consultorio) hasta el hospital u hospitales de referencia de la organización sanitaria integrada que centraliza los servicios de Atención Especializada.

En la fase inicial del estudio que ha dado lugar a este artículo, se consideró la posibilidad de incluir como variable el transporte público, desestimándose finalmente la opción de desplazamiento por este medio, ya que la frecuencia y los horarios son heterogéneas a lo largo del día y por ello, se produce un sesgo en los datos y comparabilidad de los resultados, que no se verían compensados por el plus de información resultante. Al final, se ha optado únicamente por la opción del parámetro de desplazamiento por carretera, en "automóvil" y en algún caso esporádico "caminando", cuando la cercanía para este tipo de desplazamiento precisaba menor tiempo. El análisis no incluye como condición el estado del tráfico ya que este parámetro puede variar según la hora del día y para cada uno de los días del año, con lo cual se perdería la homogeneidad imprescindible para el análisis. Por ello, lo que aquí se refleja son las condiciones teóricas ideales o estandarizadas, resultando que, si bien la distancia en el desplazamiento permanece inalterada, la duración, en circunstancias reales puede verse afectada por las condiciones de la circulación.

El análisis estadístico descriptivo se ha realizado comparativamente tanto a nivel de la totalidad de Osakidetza, Área Sanitaria ${ }^{8}$ y organizaciones sanitarias integradas, calculándose para todos ellos, la media aritmética, desviación típica y coeficiente de variación del tiempo y la distancia desde cada uno de los centros de Atención Primaria.

El análisis estadístico multivariante, se realiza a los mismos niveles que el descriptivo mediante un análisis de regresión lineal simple con el objeto de verificar, en principio, una correlación significativamente estadística, la cual tal vez nos permitiera plantear, a posteriori, la existencia de algún tipo de causalidad.

7. Buscador de centros y hospitales: https://www.osakidetza.euskadi.eus/tu-centro-sanitario-buscador/-/buscador-de-centros-sanitarios-y-hospitales/

8. Área de Salud: definida según el artículo 56 de Ley 14/1986, de 25 de abril, General de Sanidad, publicada en el BOE núm. 102, de fecha 29/04/1986: https://www.boe.es/buscar/pdf/1986/BOE-A-1986-10499-consolidado.pdf. En el caso del País Vasco cada Área de Salud se corresponde casi miméticamente con cada uno de los Territorios Históricos, salvo contadas excepciones. 
La hipótesis previa, a la hora de seleccionar las variables del estudio, es que a una mayor densidad de población en el municipio donde se enclava el centro de salud o consultorio, le debe corresponder una menor duración del desplazamiento o distancia hasta el punto donde se localiza el hospital de referencia de la organización sanitaria integrada y a la inversa. Por tanto, la variable explicativa o independiente (X) es la densidad de población y la variable explicada o dependiente $(Y)$ el tiempo del desplazamiento en minutos o en su caso la distancia cuantificada en kilómetros.

No obstante, ya desde una fase inicial de la extracción de las variables, hubo de desestimarse la densidad de población, puesto que no era un parámetro óptimo al generar una importante distorsión, evidenciada al comparar los datos municipales (población, superficie, densidad), obtenidos del Instituto Vasco de Estadística (Eustat). El resultado es que nos encontramos con municipios escasamente poblados, pero con una superficie minúscula, lo cual generaba una densidad de población más alta que otros con mayor población, si bien también más extensos:

Por ejemplo, Lanestosa (Bizkaia), tiene una población de 280 habitantes (2017) y es el municipio de menor extensión del País Vasco $\left(1,14\right.$ km²), con una densidad de población de 245,6 hab. $/ \mathrm{km}^{2}$, superior a otros más poblados, pero de mayor superficie, como Azkoitia: 11879 habitantes (2017) / 215,3 hab/km², Azpeitia: 14817 (2017) / 214,0 hab/km² o Bergara: 15013 (2017) / 198,5 hab/km², todos ellos en Gipuzkoa. En el caso contrario Vitoria (Araba/Álava), el segundo municipio más poblado del País Vasco: 242254 habitantes (2017), pero también el de mayor extensión, $276,34 \mathrm{~km}^{2}$, da como resultado una densidad de $876,7 \mathrm{hab} / \mathrm{km}^{2}$, inferior a otros municipios menos poblados.

Por ello, la variable explicativa $(X)$ seleccionada finalmente para el estudio, ha sido la población municipal, aunque resulta necesario precisar el hecho de que esta decisión tampoco está exenta de riesgo de distorsión, como luego se verá en los resultados y en la discusión.

En el análisis estadístico multivariante, las ecuaciones del método de mínimos cuadrados de la Regresión Lineal, para obtener el Coeficiente de Correlación de Pearson así como los valores (a y b) de la ecuación de la recta: $y=a+b x$, mediante las fórmulas [1], [2] y [3], son:

- Coeficiente de Correlación de Pearson $(X, Y), R=\frac{\Sigma(x-\bar{x})(y-\bar{y})}{\sqrt{\Sigma}(x-\bar{x})^{2} \Sigma(y-\bar{y})^{2}}$ [1]

- Intercepto $(a)=\frac{n \sum x y-\sum x \sum y}{n \sum x^{2}-\left(\sum x\right)^{2}}[2]$

- Pendiente de la recta $(b)=\frac{\Sigma(x-\bar{x})(y-\bar{y})}{\Sigma(x-\bar{x})^{2}}[3]$

El estudio, además, se ha complementado con un análisis de accesibilidad poblacional realizado con respecto a la media aritmética del tiempo y la distancia de los dispositivos de Atención Primaria (centro de salud, ambulatorio o consultorio), enclavados en un determinado municipio, hasta el hospital de referencia de la organización sanitaria integrada. En los escasos municipios que carecen ${ }^{9}$ de esta infraestructura, por su cercanía al centro que da servicio a otro municipio próximo, se utiliza como punto de origen las coordenadas geográficas de su casa consistorial. Se ha agrupado por intervalos temporales (isócronas) y espaciales (kilómetros), donde se indica el número de habitantes correspondiente a cada uno de ellos, el porcentaje que representan dichos habitantes en función del nivel de análisis, así como el número de municipios a los que afecta cada intervalo.

9. Se ha realizado un análisis y en Araba/Álava hay 10 municipios, en Bizkaia son 4 y en Gipuzkoa 13, del total de 251 del País Vasco, que carecen de dichos dispositivos de Atención Primaria. En todos los casos se encuentran muy próximos, tanto en tiempo como distancia, al correspondiente a otro municipio colindante o muy próximo, y algún caso, incluso, se trata de la misma trama urbana, al margen de que administrativamente sean municipios distintos. En estos casos el punto de origen de la medición hasta el hospital se ha realizado desde las coordenadas de la casa consistorial como referencia poblacional. 
El alcance del análisis de accesibilidad poblacional se refiere al País Vasco, Territorios Históricos y organizaciones sanitarias integradas, y a este respecto debemos traer a colación que existen otros estudios precedentes con los que este guarda similitud, aunque referidos a distintos ámbitos y variaciones en la metodología:

En la Región de Murcia, Palomar et al. (1996), elaboraron un estudio de accesibilidad a sus dispositivos sanitarios.

\footnotetext{
"Las mediciones se agruparon en siete intervalos, seis de ellos de 5 minutos y otro más en el que se incluyeron todas aquellas que superaban los 30 minutos. Para cada una de las zonas y áreas de salud se ha establecido el porcentaje de población incluida en cada uno de esos intervalos."
}

De naturaleza análoga a la pretensión de nuestro artículo también hay otros que, mediante el uso de las isócronas correspondientes a intervalos de tiempo de idéntica duración, les han atribuido una valoración cualitativa al grado de accesibilidad temporal de un determinado servicio. En un estudio de accesibilidad de la población rural a los servicios básicos de salud, de la provincia de Teruel, Escalona y Díez, (2003), establecen cuatro intervalos o categorías de accesibilidad:

- $A_{i}=f\left(c_{i j}\right)$, con $c_{i j}<15$ minutos: accesibilidad óptima.

- $A_{i}=f\left(c_{i j}\right)$, con $16<c_{i j}<30$ minutos: accesibilidad favorable/aceptable.

- $A_{i}=f\left(c_{i j}\right)$, con $31<c_{i j}<45$ minutos: accesibilidad desfavorable.

$-A_{i}=f\left(c_{i j}\right)$, con $c_{i j}>46$ minutos: accesibilidad muy desfavorable.

En otro análisis, realizado por Rodríguez (2011), también de accesibilidad geográfica de la población a los hospitales de Alta Resolución de Andalucía se utilizan los mismos intervalos de tiempo.

En Castilla la Mancha se han dado a conocer datos referidos a la accesibilidad hasta los Centros de Salud: "Sanidad cumple con distancia máxima de 30 minutos a todos los centros de Salud tras cotejar datos con Fomento", (Infosalus.com, 2013). En la Comunidad Autónoma de Extremadura, la Unidad Docente de Medicina de Familia y Comunitaria de Mérida, en la descripción del Área Sanitaria cuantifica la accesibilidad geográfica de sus recursos sanitarios: "La distancia máxima al Hospital es de 78 km. Y en las isócronas el tiempo medio de acceso es menor de 20 minutos".

En nuestro caso, para el estudio en Osakidetza, el formato de presentación lo hemos dividido en siete intervalos, de forma comparable a trabajos precedentes, siendo la accesibilidad temporal en rangos de cinco minutos cada uno, de 0 hasta más de 30 minutos, redondeados al minuto y la distancia, en rangos de cinco kilómetros cada uno, de 0 hasta más de $30 \mathrm{~km}$., redondeados a un solo decimal.

De la misma forma que otros artículos, en base a los datos cuantitativos sobre accesibilidad, se pronuncian emitiendo un juicio de valor cualitativo (accesibilidad óptima, favorable, desfavorable y muy desfavorable), en el presente artículo también haremos una valoración, correspondiente a nuestros siete intervalos de accesibilidad temporal y espacial:

\section{RESULTADOS Y DISCUSIÓN}

Una vez procesados los datos, se detallarán a continuación y en el anexo del artículo, los resultados obtenidos, que serán objeto de comentario y discusión, fruto de los cuales se extraerán las oportunas conclusiones.

En primer lugar, una vez aplicada la metodología, el tabla 3, resume los cálculos estadísticos descriptivos (media aritmética, desviación típica y coeficiente de variación), del tiempo y distancia desde el centro de Atención Primaria hasta el hospital de referencia, tanto a nivel provincial como de organización sanitaria integrada; el tabla 4 hace lo mismo, mediante un análisis estadístico multivariante. Estos parámetros también nos servirán para estimar la equidad espacial y la eficiencia, e incluso la potencialidad de mejora de las infraestructuras hasta los hospitales. 
El análisis de accesibilidad poblacional (tabla 5), se ha realizado con los rangos de tiempo y distancia municipales, señalados en el tabla 2 y luego, para una mejor representación gráfica, se ha plasmado sobre el mapa 3, a nivel comunidad autónoma con demarcaciones municipales y todos los centros de Atención Primaria, con la indicación de una letra desde la A hasta la $\mathrm{H}$, indicativo de la organización sanitaria integrada correspondientes a la leyenda del mapa y sus hospitales de referencia, representados por el icono característico y en los mapas 4 (tiempo) y 5 (distancia).

Tabla 2. Accesibilidad Temporal y Espacial.

\begin{tabular}{|c|c|}
\hline \multicolumn{2}{|c|}{ ACCESIBILIDAD TEMPORAL } \\
\hline Entre 0 y 5 minutos & Accesibilidad óptima. \\
\hline De 6 a 10 minutos & Accesibilidad muy favorable. \\
\hline De 11 a 15 minutos & Accesibilidad favorable. \\
\hline De 16 a 20 minutos & Accesibilidad normal. \\
\hline De 21 a 25 minutos & Accesibilidad desfavorable. \\
\hline De 26 a 30 minutos & Accesibilidad muy desfavorable. \\
\hline Más de 30 minutos & Accesibilidad inaceptable. \\
\hline \multicolumn{2}{|c|}{ ACCESIBILIDAD ESPACIAL } \\
\hline Entre 0 y 5 km. & Distancia óptima. \\
\hline Más de 5 y hasta $10 \mathrm{~km}$. & Distancia muy favorable. \\
\hline Más de 10 y hasta 15 km. & Distancia favorable. \\
\hline Más de 15 y hasta 20 km. & Distancia normal. \\
\hline Más de 20 y hasta 25 km. & Distancia desfavorable. \\
\hline Más de 25 y hasta 30 km. & Distancia muy desfavorable. \\
\hline Más de 30 km. & Distancia inaceptable. \\
\hline
\end{tabular}

Fuente: Elaboración propia.

Tabla 3. Resumen Tiempo/Distancia desde dispositivos de Atención Primaria (Descriptivo).

\begin{tabular}{|c|c|c|c|c|c|c|}
\hline \multirow{2}{*}{ OSAKIDETZA } & \multicolumn{2}{|c|}{ Media Aritmética } & \multicolumn{2}{|c|}{ Desviación Típica } & \multicolumn{2}{|c|}{ Coeficiente Variación } \\
\hline & $\begin{array}{l}\text { Tiempo } \\
\text { (Minutos) }\end{array}$ & $\begin{array}{c}\text { Distancia } \\
\text { (Kilómetros) }\end{array}$ & $\begin{array}{l}\text { Tiempo } \\
\text { (Minutos) }\end{array}$ & $\begin{array}{c}\text { Distancia } \\
\text { (Kilómetros) }\end{array}$ & $\begin{array}{c}\text { Tiempo } \\
(\%)\end{array}$ & $\begin{array}{c}\text { Distancia } \\
(\%)\end{array}$ \\
\hline TOTAL OSAKIDETZA & 17,06 & 13,87 & 9,48 & 11,01 & $55,59 \%$ & $79,35 \%$ \\
\hline
\end{tabular}




\begin{tabular}{|l|c|c|c|c|c|c|}
\hline \multirow{2}{*}{ AREA ARABA } & \multicolumn{2}{|c|}{ Media Aritmética } & \multicolumn{2}{c|}{ Desviación Típica } & \multicolumn{2}{c|}{ Coeficiente Variación } \\
\cline { 2 - 7 } & $\begin{array}{c}\text { Tiempo } \\
\text { (Minutos) }\end{array}$ & $\begin{array}{c}\text { Distancia } \\
\text { (Kilómetros) }\end{array}$ & $\begin{array}{c}\text { Tiempo } \\
\text { (Minutos) }\end{array}$ & $\begin{array}{c}\text { Distancia } \\
\text { (Kilómetros) }\end{array}$ & $\begin{array}{c}\text { Tiempo } \\
(\%)\end{array}$ & $\begin{array}{c}\text { Distancia } \\
(\%)\end{array}$ \\
\hline Araba & 22,05 & 18,17 & 11,17 & 13,83 & $50,67 \%$ & $76,09 \%$ \\
\hline $\begin{array}{l}\text { Arabako Errioxa- } \\
\text { Rioja Alavesa }\end{array}$ & 15,78 & 13,19 & 6,73 & 7,58 & $42,65 \%$ & $57,44 \%$ \\
\hline TOTAL AREA & 20,94 & 17,29 & 10,79 & 13,08 & $51,54 \%$ & $75,66 \%$ \\
\hline
\end{tabular}

\begin{tabular}{|l|c|c|c|c|c|c|}
\hline \multicolumn{1}{|c|}{ AREA BIZKAIA } & \multicolumn{2}{|c|}{ Media Aritmética } & \multicolumn{2}{c|}{ Desviación Típica } & \multicolumn{2}{c|}{ Coeficiente Variación } \\
\cline { 2 - 7 } & $\begin{array}{c}\text { Tiempo } \\
\text { (Minutos) }\end{array}$ & $\begin{array}{c}\text { Distancia } \\
\text { (Kilómetros) }\end{array}$ & $\begin{array}{c}\text { Tiempo } \\
\text { (Minutos) }\end{array}$ & $\begin{array}{c}\text { Distancia } \\
\text { (Kilómetros) }\end{array}$ & $\begin{array}{c}\text { Tiempo } \\
(\%)\end{array}$ \\
\hline Barakaldo-Sestao & 6,70 & 2,38 & 2,05 & 1,36 & $30,62 \%$ & $57,30 \%$ \\
\hline Barrualde-Galdakao & 18,08 & 16,55 & 9,26 & 10,76 & $51,19 \%$ & $65,01 \%$ \\
\hline Bilbao-Basurto & 11,00 & 4,54 & 3,14 & 2,52 & $28,53 \%$ & $55,58 \%$ \\
\hline Ezkerraldea-Enkarte- & 25,14 & 23,38 & 13,30 & 15,36 & $52,92 \%$ & $65,72 \%$ \\
\hline rri-Cruces & 16,91 & 12,23 & 5,80 & 5,84 & $34,28 \%$ & $47,69 \%$ \\
\hline Uribe & 17,32 & 14,24 & 9,92 & 11,72 & $57,29 \%$ & $82,27 \%$ \\
\hline TOTAL AREA & & & & & 5 \\
\hline
\end{tabular}

\begin{tabular}{|c|c|c|c|c|c|c|}
\hline \multirow{2}{*}{ AREA GIPUZKOA } & \multicolumn{2}{|c|}{ Media Aritmética } & \multicolumn{2}{|c|}{ Desviación Típica } & \multicolumn{2}{|c|}{ Coeficiente Variación } \\
\hline & $\begin{array}{l}\text { Tiempo } \\
\text { (Minutos) }\end{array}$ & $\begin{array}{c}\text { Distancia } \\
\text { (Kilómetros) }\end{array}$ & $\begin{array}{l}\text { Tiempo } \\
\text { (Minutos) }\end{array}$ & $\begin{array}{c}\text { Distancia } \\
\text { (Kilómetros) }\end{array}$ & $\begin{array}{l}\text { Tiempo } \\
\text { (\%) }\end{array}$ & $\begin{array}{l}\text { Distancia } \\
\quad(\%)\end{array}$ \\
\hline Donostialdea & 13,88 & 11,41 & 6,83 & 9,46 & $49,17 \%$ & $82,92 \%$ \\
\hline Goierri-Alto Urola & 19,95 & 16,76 & 8,33 & 7,10 & $41,74 \%$ & $42,39 \%$ \\
\hline Bidasoa & 7,50 & 3,00 & 4,56 & 1,94 & $60,74 \%$ & $64,59 \%$ \\
\hline $\begin{array}{l}\text { Bajo Deba (Debaba- } \\
\text { rrena) }\end{array}$ & 15,30 & 12,08 & 5,64 & 5,36 & $36,86 \%$ & $44,37 \%$ \\
\hline Alto Deba & 13,90 & 9,96 & 4,46 & 4,07 & $32,09 \%$ & $40,83 \%$ \\
\hline Tolosaldea & 12,35 & 8,93 & 4,47 & 4,45 & $36,22 \%$ & $49,85 \%$ \\
\hline TOTAL AREA & 14,66 & 11,51 & 6,98 & 7,54 & $47,57 \%$ & $65,48 \%$ \\
\hline
\end{tabular}

Fuente: Elaboración propia, a partir de datos de Osakidetza y Google Earth. 
Tabla 4. Resumen Tiempo/Distancia desde dispositivos de Atención Primaria (Multivariante).

\begin{tabular}{|c|c|c|c|c|c|c|c|c|c|c|}
\hline \multirow[b]{2}{*}{ AREA ARABA } & \multicolumn{5}{|c|}{ Analisis Regresión (X = Población; $Y$ = Tiempo) } & \multicolumn{5}{|c|}{ Analisis Regresión ( $\mathrm{X}=$ Población; $\mathrm{Y}=$ Distancia) } \\
\hline & a & b & $\mathrm{R}$ & R2 & $\begin{array}{c}\% \text { Explicativo } \\
\left(1-R^{2}\right)\end{array}$ & a & $b$ & $\mathrm{R}$ & $\mathrm{R} 2$ & $\begin{array}{c}\% \text { Explicativo } \\
\left(1-R^{2}\right)\end{array}$ \\
\hline Araba & 29,08909 & $-0,00008$ & $-0,83213$ & 0,69243 & $69,243 \%$ & 26,94206 & $-0,00010$ & $-0,83750$ & 0,70140 & $70,140 \%$ \\
\hline $\begin{array}{l}\text { Arabako Errioxa-Rioja } \\
\text { Alavesa }\end{array}$ & 13,59365 & 0,00199 & 0,27937 & 0,07804 & $7,804 \%$ & 10,41425 & 0,00252 & 0,31524 & 0,09938 & $9,938 \%$ \\
\hline TOTAL AREA & 25,76021 & $-0,00007$ & $-0,67905$ & 0,46112 & $46,112 \%$ & 23,50695 & $-0,00009$ & $-0,72246$ & 0,52194 & $52,194 \%$ \\
\hline
\end{tabular}

\begin{tabular}{|c|c|c|c|c|c|c|c|c|c|c|}
\hline \multirow[b]{2}{*}{ AREA ARABA } & \multicolumn{5}{|c|}{ Analisis Regresión (X = Población; $Y$ = Tiempo) } & \multicolumn{5}{|c|}{ Analisis Regresión (X = Población; $Y$ = Distancia) } \\
\hline & a & b & $\mathrm{R}$ & R2 & $\begin{array}{c}\text { \% Explicativo } \\
\left(1-R^{2}\right)\end{array}$ & a & $\mathrm{b}$ & $\mathrm{R}$ & $\mathrm{R} 2$ & $\begin{array}{c}\text { \% Explicativo } \\
\left(1-R^{2}\right)\end{array}$ \\
\hline Barakaldo-Sestao & 8,20133 & $-0,00002$ & $-0,30842$ & 0,09512 & $9,512 \%$ & 4,53363 & $-0,00003$ & $-0,66569$ & 0,44315 & $44,315 \%$ \\
\hline Barrualde-Galdakao & 19,63285 & $-0,00024$ & $-0,26575$ & 0,07062 & $7,062 \%$ & 18,00645 & $-0,00023$ & $-0,21511$ & 0,04627 & $4,627 \%$ \\
\hline Bilbao-Basurto & 9,93018 & 0,00000 & 0,09182 & 0,00843 & $0,843 \%$ & 8,09623 & $-0,00001$ & $-0,38020$ & 0,14455 & $14,455 \%$ \\
\hline $\begin{array}{l}\text { Ezkerraldea-Enkarte- } \\
\text { rri-Cruces }\end{array}$ & 29,87965 & $-0,00028$ & $-0,47461$ & 0,22526 & $22,526 \%$ & 28,91968 & $-0,00033$ & $-0,48049$ & 0,23087 & $23,087 \%$ \\
\hline Uribe & 17,29897 & $-0,00003$ & $-0,11271$ & 0,01270 & $1,270 \%$ & 12,76589 & $-0,00004$ & $-0,15151$ & 0,02295 & $2,295 \%$ \\
\hline TOTAL AREA & 18,96369 & $-0,00003$ & $-0,31646$ & 0,10015 & $10,015 \%$ & 16,73436 & $-0,00004$ & $-0,40505$ & 0,16406 & $16,406 \%$ \\
\hline
\end{tabular}

\begin{tabular}{|c|c|c|c|c|c|c|c|c|c|c|}
\hline \multirow[b]{2}{*}{ AREA ARABA } & \multicolumn{5}{|c|}{ Analisis Regresión (X = Población; Y = Tiempo) } & \multicolumn{5}{|c|}{ Analisis Regresión (X = Población; Y = Distancia) } \\
\hline & a & b & $\mathrm{R}$ & R2 & $\begin{array}{l}\% \text { Explicativo } \\
\left(1-R^{2}\right)\end{array}$ & a & $\mathrm{b}$ & $\mathrm{R}$ & R2 & $\begin{array}{c}\text { \% Explicativo } \\
\left(1-\mathrm{R}^{2}\right)\end{array}$ \\
\hline Donostialdea & 17,76466 & $-0,00005$ & $-0,56714$ & 0,32165 & $32,165 \%$ & 16,54947 & $-0,00007$ & $-0,56311$ & 0,31709 & $31,709 \%$ \\
\hline Goierri-Alto Urola & 19,03526 & 0,00018 & 0,11118 & 0,01236 & $1,236 \%$ & 16,83900 & $-0,00002$ & $-0,01197$ & 0,00014 & $0,014 \%$ \\
\hline Bidasoa & 4,79059 & 0,00007 & 0,32929 & 0,10843 & $10,843 \%$ & 2,00655 & 0,00003 & 0,28383 & 0,08056 & $8,056 \%$ \\
\hline $\begin{array}{l}\text { Bajo Deba (Debaba- } \\
\text { rrena) }\end{array}$ & 14,41039 & 0,00008 & 0,13912 & 0,01935 & $1,935 \%$ & 10,73266 & 0,00013 & 0,22097 & 0,04883 & $4,883 \%$ \\
\hline Alto Deba & 16,54031 & $-0,00033$ & $-0,52892$ & 0,27976 & $27,976 \%$ & 12,73637 & $-0,00035$ & $-0,60997$ & 0,37207 & $37,207 \%$ \\
\hline Tolosaldea & 12,91932 & $-0,00019$ & $-0,23329$ & 0,05442 & $5,442 \%$ & 9,66805 & $-0,00025$ & $-0,30141$ & 0,09085 & $9,085 \%$ \\
\hline TOTAL AREA & 15,73276 & $-0,00004$ & $-0,30232$ & 0,09140 & $9,140 \%$ & 12,78712 & $-0,00005$ & $-0,33289$ & 0,11081 & $11,081 \%$ \\
\hline TOTAL OSAKIDETZA & 18,69371 & $-0,00003$ & $-0,33653$ & 0,11325 & $11,325 \%$ & 16,23678 & $-0,00005$ & $-0,41949$ & 0,17597 & $17,597 \%$ \\
\hline
\end{tabular}

Fuente: Elaboración propia, a partir de datos de Osakidetza y Google Earth. 


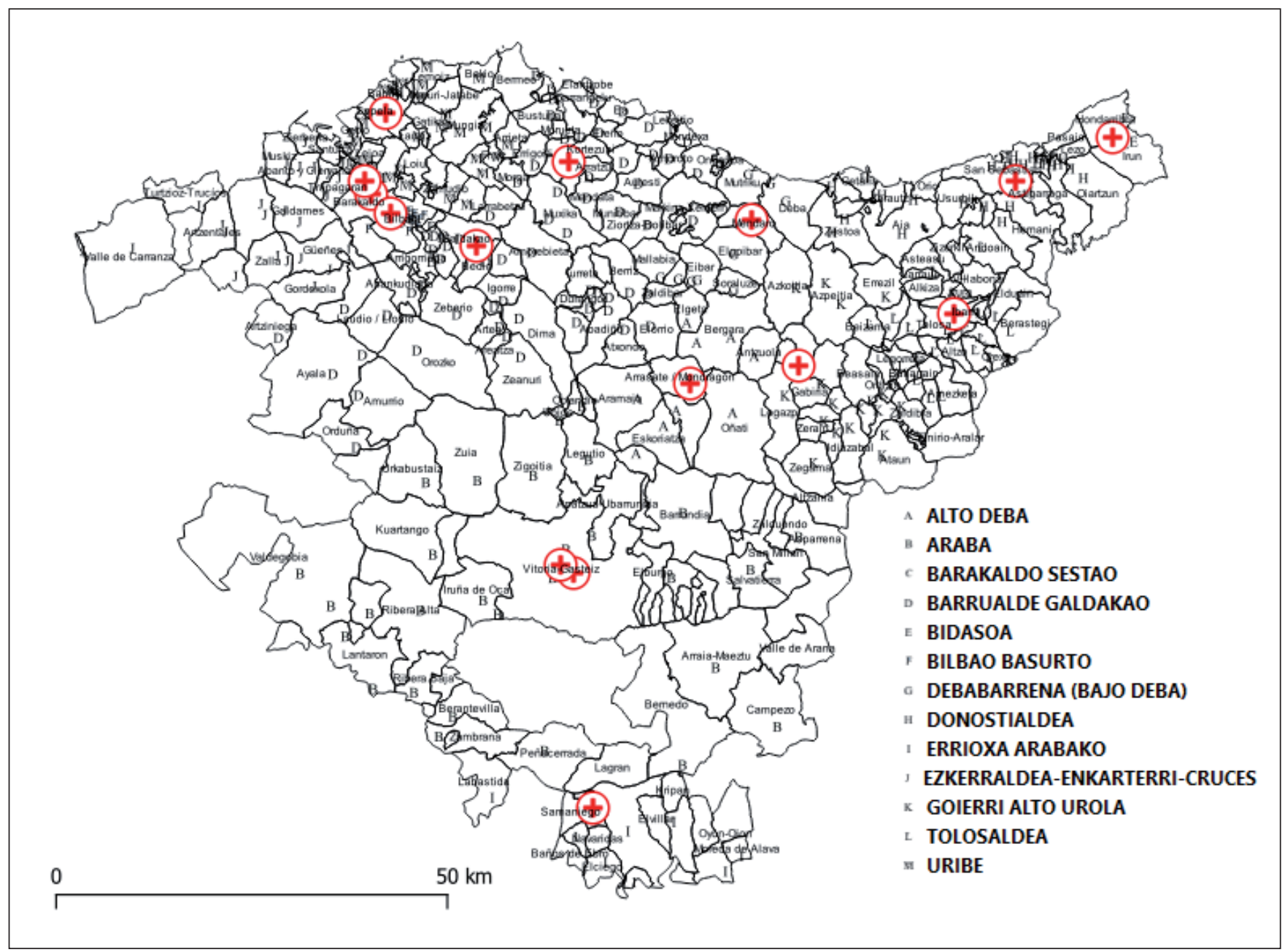

Mapa 3. Centros del País Vasco. Fuente: Elaboración propia, a partir de datos de Osakidetza y Google Earth.

Tabla 5. Accesibilidad población del País Vasco, por intervalos ${ }^{10}$.

\begin{tabular}{|c|c|c|c|c|c|c|c|}
\hline \multicolumn{8}{|c|}{ EUSKADI } \\
\hline TIEMPO & Municipios & Población & $\%$ & DISTANCIA & Municipios & Población & $\%$ \\
\hline Entre 0 y 5 minutos & 10 & 52.354 & $2,41 \%$ & Entre 0 y 5 km. & 34 & 959.831 & $44,11 \%$ \\
\hline De 6 a 10 minutos & 38 & 799.946 & $36,77 \%$ & Más de 5 y hasta 10 km. & 51 & 534.037 & $24,54 \%$ \\
\hline De 11 a 15 minutos & 58 & 771.943 & $35,48 \%$ & Más de 10 y hasta $15 \mathrm{~km}$. & 53 & 324.402 & $14,91 \%$ \\
\hline De 16 a 20 minutos & 56 & 328.572 & $15,10 \%$ & Más de 15 y hasta 20 km. & 38 & 124.431 & $5,72 \%$ \\
\hline De 21 a 25 minutos & 32 & 96.914 & $4,45 \%$ & Más de 20 y hasta $25 \mathrm{~km}$. & 28 & 129.325 & $5,94 \%$ \\
\hline De 26 a 30 minutos & 30 & 75.316 & $3,46 \%$ & Más de 25 y hasta 30 km. & 18 & 28.199 & $1,30 \%$ \\
\hline Más de 30 minutos & 27 & 50.774 & $2,33 \%$ & Más de 30 km. & 29 & 75.594 & $3,47 \%$ \\
\hline TOTAL & 251 & 2.175 .819 & $100,00 \%$ & TOTAL & 251 & 2.175 .819 & $100,00 \%$ \\
\hline
\end{tabular}

Fuente: Elaboración propia Osakidetza, Eustat y Google Earth.

10. El Anexo final incluye tablas de accesibilidad similares, a nivel provincial y de organización sanitaria. 


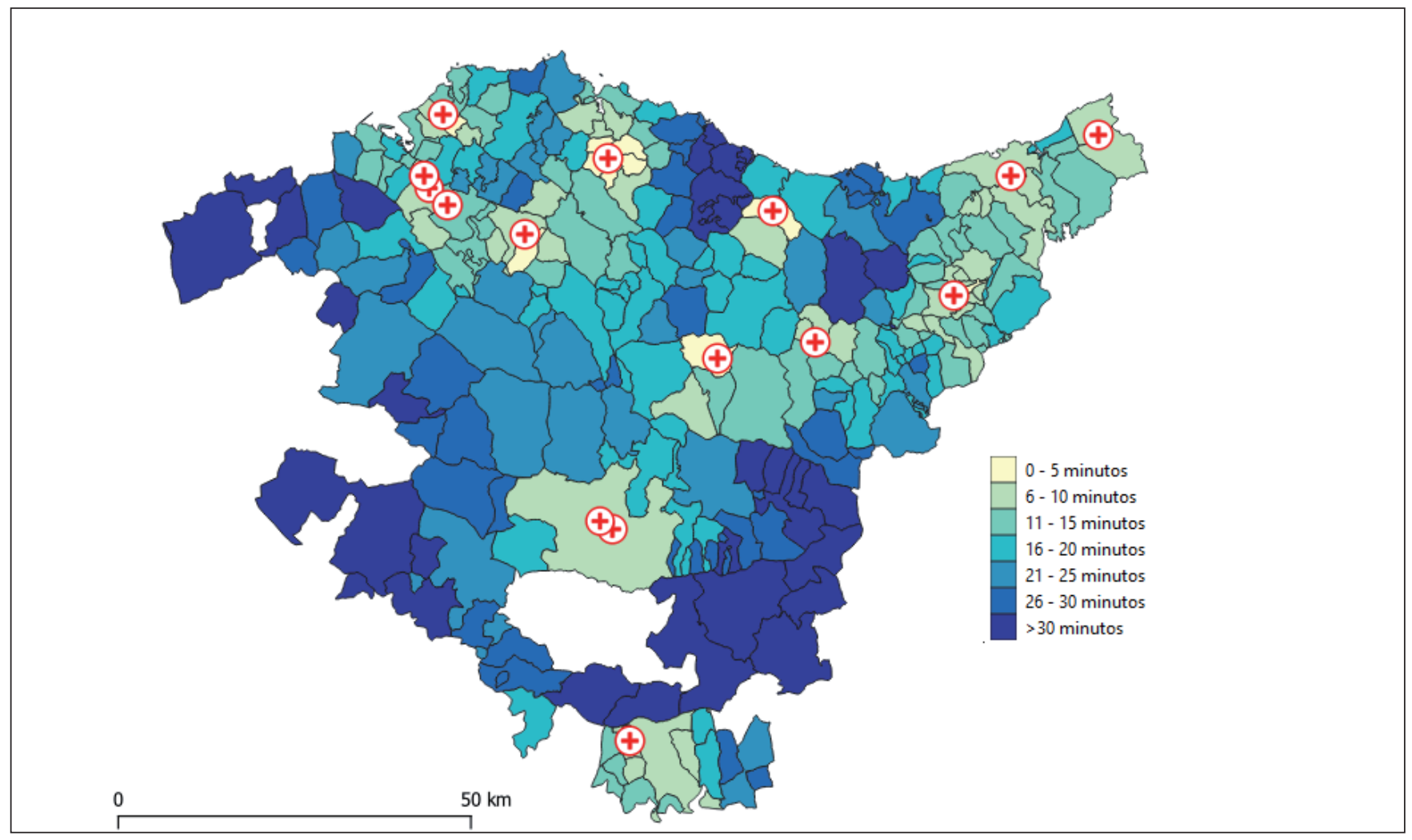

Mapa 4. Accesibilidad hospitalaria desde municipios en función del tiempo (minutos). Fuente: Elaboración propia, a partir de datos de Osakidetza y Google Earth.

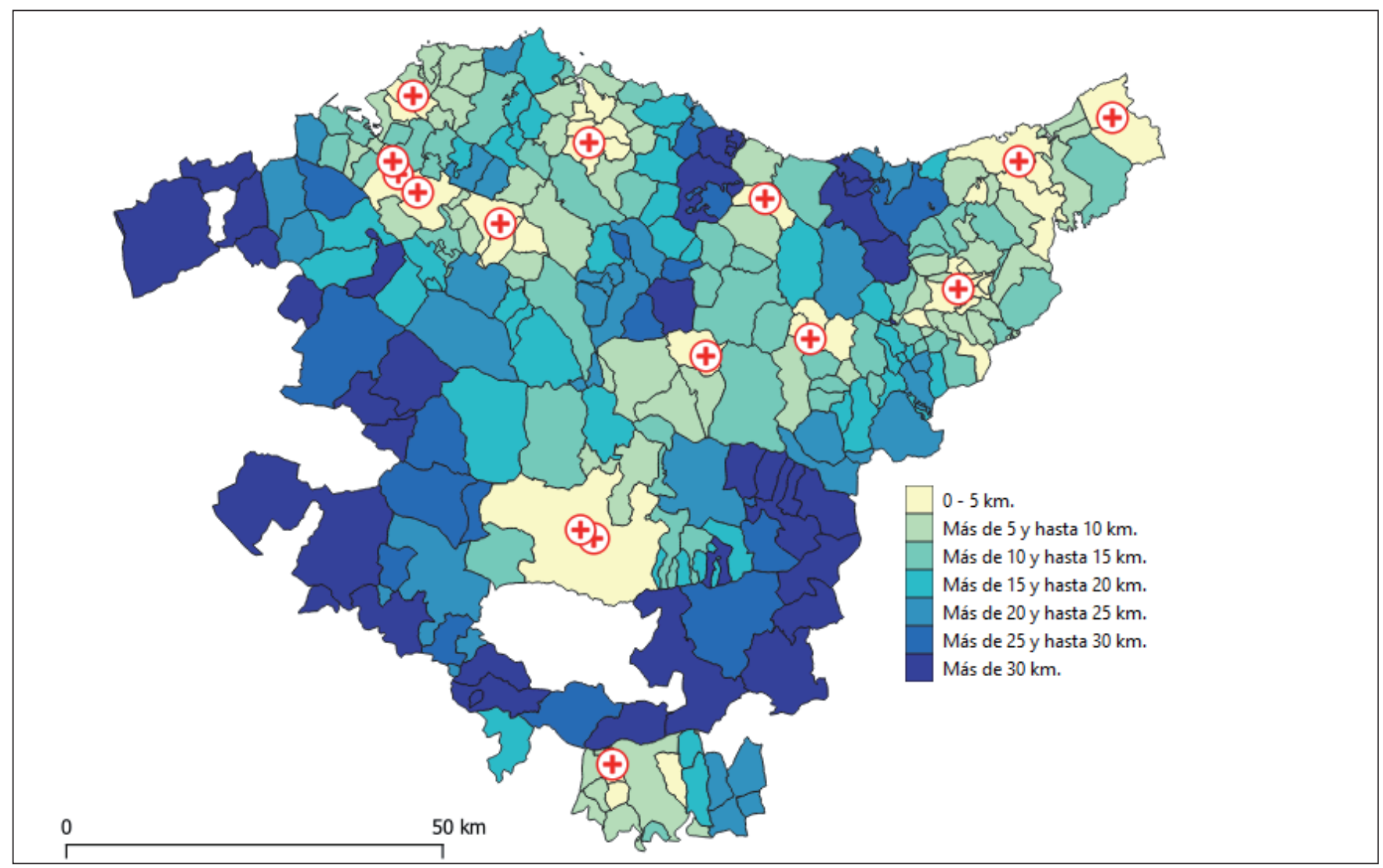

Mapa 5. Accesibilidad hospitalaria desde municipios en función de la distancia (km.). Fuente: Elaboración propia, a partir de datos de Osakidetza y Google Earth. 
Para relativizar y contextualizar los parámetros de tiempo y distancia de los mapas anteriores con el tamaño de la población municipal (eficiencia vs. equidad), se ha incluido el siguiente mapa 6.

Este ha segmentado en rangos, el tamaño de población municipal, cuya elección responde al principio de Pareto. Dicho principio empírico, habitual en el ámbito de las Ciencias Sociales y también llamado "Regla del 80 / 20", simplificando, viene a significar, que, aproximadamente el $80 \%$ de los efectos, responde al $20 \%$ de las causas, o viceversa.

El rango que incluye la población de todos los municipios con más de 10.000 habitantes, que son 42, suman 1.742 .965 habitantes $(80,11 \%$ de la población total del País Vasco). Estos 42 municipios, con respecto al total de 251, suponen el 16,73\%. El resto de municipios con población inferior a 10.000 habitantes (209), suponen el 83,26 del total de municipios, pero sólo el 19,89\% de su población. Estos 209 municipios menos poblados, se han segmentado, a su vez, en otros cuatro rangos, para una mejor visualización; donde es posible apreciar que los hospitales se asientan habitualmente en los municipios más poblados, los cuales, en zonas rurales, son también cabeceras de comarca.

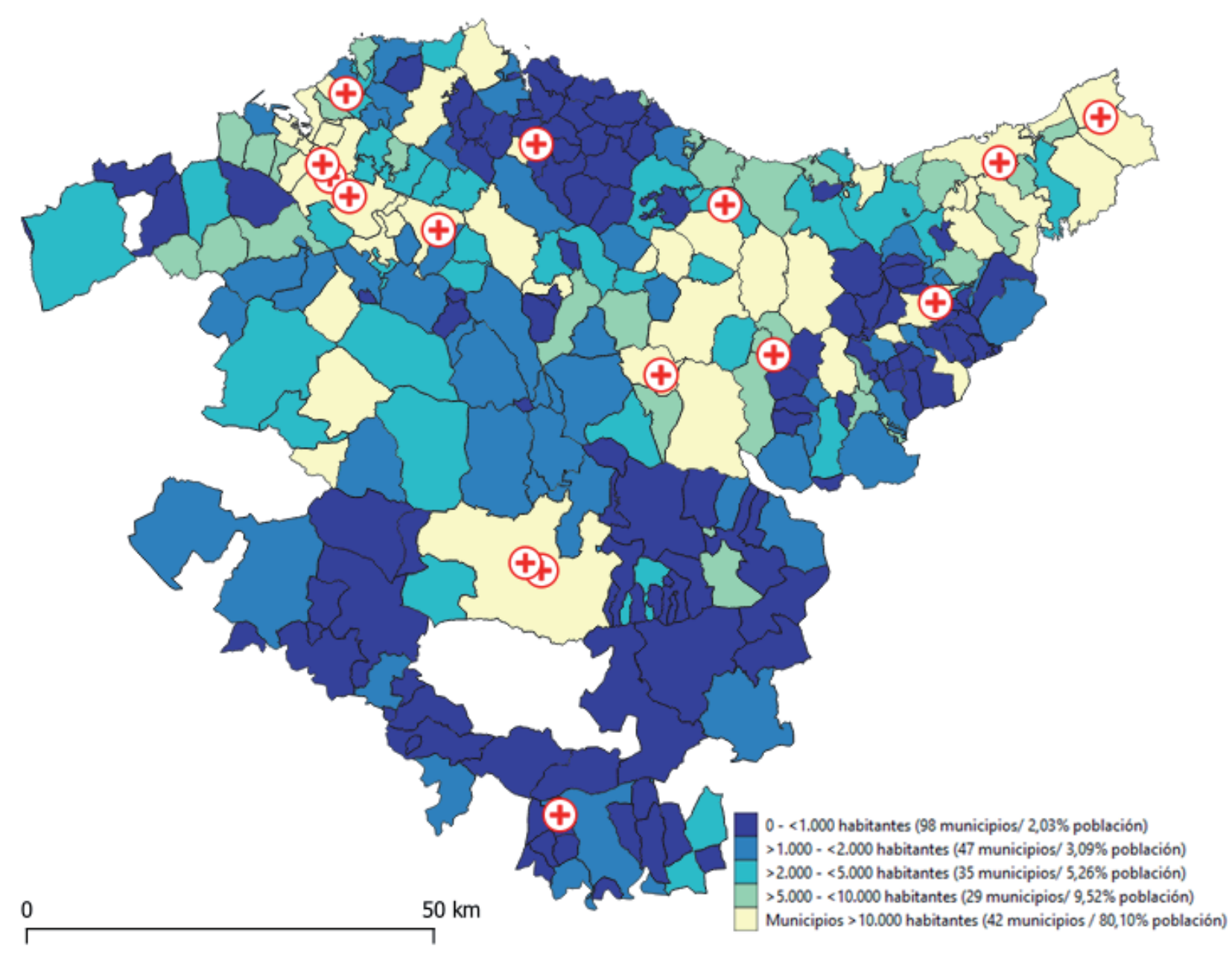

Mapa 6. Rangos de tamaño poblacional municipal. Fuente: Elaboración propia, a partir de datos de Osakidetza, Eustat y Google Earth.

Vistos los resultados, la discusión debe focalizarse en si se evidencia el objetivo primario desde el punto de vista de la equidad espacial y la eficiencia, a la hora de distribuir geográficamente los recursos asistenciales y 
si son compatibles con las necesidades de la población. A primera vista, se aprecia que el resultado en todos los casos es muy favorable, tanto a nivel de Osakidetza como de Área Sanitaria; es mejor, en tanto en cuanto menores sean la distancia y sobre el todo el tiempo, promediados, necesarios para acceder al hospital. Tanto en el caso de la equidad como de la eficiencia, el parámetro más importante es el tiempo de acceso, siendo la distancia secundaria. No obstante, ambos datos combinados pueden servir para estimar el margen de mejora mediante la intervención en las vías de comunicación, como luego se explicará.

Aunque las diferencias entre áreas no son significativas, el mejor resultado lo obtenemos en Gipuzkoa, la cual en los dos parámetros tiene una media aritmética inferior, seguida de Bizkaia y luego Araba/Álava. Esto se puede interpretar como que un menor tiempo/distancia de acceso, implica una mayor eficiencia desde el punto de vista geográfico y viceversa.

Si lo que se busca en el análisis es la equidad, hay que fijarse en el coeficiente de variación y la interpretación que podemos hacer, en este caso, es que, a un menor coeficiente de variación de los parámetros analizados, debe inferirse un mayor grado de equidad geográfica. También en este caso los resultados son mejores en el Área de Gipuzkoa, pero ahora es Araba/Álava quien ocupa la segunda posición y Bizkaia, es desde este punto de vista, la menos equitativa. Resumidamente la situación de las Áreas Sanitarias se puede expresar en forma de prelación de mejor a peor de la siguiente forma:

Tabla 6. Comparación eficiencia vs. equidad por Área Sanitaria.

\begin{tabular}{|c|l|l|l|l|l|l|c|}
\hline \multicolumn{2}{|c|}{ AREA SANITARIA } \\
\hline \multicolumn{2}{|c|}{ EFICIENCIA } \\
\hline \multicolumn{2}{|c|}{ Tiempo } & \multicolumn{2}{c|}{ Distancia } & \multicolumn{3}{c|}{ Tiempo } \\
\hline 1 & Gipuzkoa & Gipuzkoa & 1 & 1 & Gipuzkoa & Gipuzkoa & 1 \\
\hline 2 & Bizkaia & Bizkaia & 2 & 2 & Araba & Araba & 2 \\
\hline 3 & Araba & Araba & 3 & 3 & Bizkaia & Bizkaia & 3 \\
\hline
\end{tabular}

Fuente: Elaboración propia, a partir de datos de Osakidetza y Google Earth.

El resultado tiene buena parte de lógica ya que el Área de Gipuzkoa es la de menor extensión territorial y a la vez, la que dispone de un mayor número de organizaciones sanitarias integradas, repartidas de forma muy homogénea por todo el territorio provincial, tal como se aprecia en los mapas precedentes. También el hospital correspondiente, en todos los casos, se encuentra centrado en relación al espacio geográfico de cobertura de las organizaciones sanitarias integradas guipuzcoanas. En el caso de las Áreas Sanitarias de Bizkaia y Araba/Álava, ambas de mayor extensión y menor número de organizaciones sanitarias integradas, los hospitales se encuentran, en general, menos centrados en el territorio, por lo que el resultado es una distribución mucho más heterogénea, lo cual se traduce un coeficiente de variación superior.

Cuando se analiza el gradiente o la diferencia de proporción entre el coeficiente de variación del tiempo y la distancia dentro de un Área Sanitaria u organización sanitaria integrada, en todos los casos es más alto el correspondiente a la distancia, incluso considerable, en algunos casos.

Pensamos que esta variación puede deberse al diferente estado o calidad de las vías de comunicación, autovías o carreteras etc., de tal forma que en algún caso, aunque el coeficiente de variación de la distancia es elevado, la mismo ratio cuando se trata del tiempo, ya no los es tanto. La interpretación más plausible, cuando el coeficiente de variación del tiempo es notablemente menor pese a la distancia, se debe a que el recorrido desde el centro de Atención Primaria hasta el hospital de referencia, se realiza por vías que permiten mayores velocidades de circulación o la orografía es más favorable. Por lo que cuando el caso sea el contrario (gradientes más reducidos entre ambos coeficientes), quizás sea posible actuar sobre las infraestructuras para mejorar la accesibilidad temporal. 
Tabla 7. Comparación eficiencia vs. equidad por organización sanitaria integrada.

\begin{tabular}{|c|c|c|c|c|c|c|c|}
\hline \multicolumn{8}{|c|}{ ORGANIZACIONES SANITARIAS INTEGRADAS } \\
\hline \multicolumn{4}{|c|}{ EFICIENCIA } & \multicolumn{4}{|c|}{ EQUIDAD } \\
\hline \multicolumn{2}{|r|}{ Tiempo } & \multicolumn{2}{|c|}{ Distancia } & \multicolumn{2}{|r|}{ Tiempo } & \multicolumn{2}{|c|}{ Distancia } \\
\hline 1 & Barakaldo-Sestao & Barakaldo-Sestao & 1 & 1 & Bilbao-Basurto & Alto Deba & 1 \\
\hline 2 & Bidasoa & Bidasoa & 2 & 2 & Barakaldo-Sestao & Goierri-Alto Urola & 2 \\
\hline 3 & Bilbao-Basurto & Bilbao-Basurto & 3 & 3 & Alto-Deba & Debabarrena & 3 \\
\hline 4 & Tolosaldea & Tolosaldea & 4 & 4 & Uribe & Uribe & 4 \\
\hline 5 & Donostialdea & Alto-Deba & 5 & 5 & Tolosaldea & Tolosaldea & 5 \\
\hline 6 & Alto-Deba & Donostialdea & 6 & 6 & Debabarrena & Bilbao-Basurto & 6 \\
\hline 7 & Debabarrena & Debabarrena & 7 & 7 & Goierri-Alto Urola & Barakaldo-Sestao & 7 \\
\hline 8 & Arabako-Errioxa & Uribe & 8 & 8 & Arabako-Errioxa & Arabako-Errioxa & 8 \\
\hline 9 & Uribe & Arabako-Errioxa & 9 & 9 & Donostialdea & Bidasoa & 9 \\
\hline 10 & Barrualde-Galdako & Barrualde-Galdako & 10 & 10 & Araba & Barrualde-Galdakao & 10 \\
\hline 11 & Goierri-Alto Urola & Goierri-Alto Urola & 11 & 11 & Barrualde-Galdakao & Ezkerraldea-Enkart. & 11 \\
\hline 12 & Araba & Araba & 12 & 12 & Ezkerraldea-Enkart. & Araba & 12 \\
\hline 13 & Ezkerraldea-Enkart. & Ezkerraldea-Enkart. & 13 & 13 & Bidasoa & Donostialdea & 13 \\
\hline
\end{tabular}

Fuente: Elaboración propia, a partir de datos de Osakidetza y Google Earth.

Cuando el análisis se hace a nivel de organización sanitaria integrada, las diferencias entre unas y otras son notables, reflejando la siguiente prelación, de mejor a peor, de la cual no es necesaria mayor explicación para su interpretación ya que resulta evidente y refleja de manera clara los parámetros analizados y la dispersión de los datos.

En cuanto al elevado coeficiente de variación, referido al tiempo de acceso para la organización sanitaria integrada Bidasoa, si bien comparativamente con otras es muy elevado, consideramos no es significativo ya que en términos absolutos sigue siendo muy reducido: la media aritmética, es en este caso 7,50 minutos y la desviación típica oscila $\pm 4,56$ minutos en torno a dicha cifra, reducida, en cualquier caso, por lo que no es digno de consideración a los efectos de cuantificar la inequidad.

Del análisis estadístico multivariante podemos concluir que en términos generales se cumple la hipótesis de que a una mayor población municipal del lugar donde se encuentra el centro de Atención Primaria, le corresponde un menor tiempo o distancia hasta su hospital de referencia, puesto que tanto a nivel de Osakidetza, Área Sanitara y la mayoría de las organizaciones sanitarias integradas la correlación es negativa. Esta es una medida de detección de la equidad, ya que un resultado peor en accesibilidad, solo tiene justificación cuando la población resulta más escasa. Todo ello en aras del equilibrio entre la equidad y el principio de sostenibilidad económica, mediante la aplicación y el uso eficiente de los recursos sanitarios.

No obstante, no se evidencia una alta correlación negativa, salvo en el Área de Araba/Álava en general y en la organización sanitaria integrada del mismo nombre, en particular: En todos los casos analizados, sin excepción, al tratar de plasmar gráficamente los datos en un diagrama de dispersión (X,Y) no se obtiene una imagen representativa que permita identificar tendencia evidente alguna debido a las diferencias de escala poblacional, puesto que algún municipio cuenta con varios miles o cientos de miles de habitantes y otros, son escasamente un ciento. 
En el caso más evidente de correlación negativa, la organización sanitaria integrada Araba/Álava, el valor de la R de Pearson, para el análisis del tiempo, es -0,832 y la ecuación de la recta y = 29,089 - 0,00008x. Esto se puede interpretar como que, al partir de un valor inicial de 29,089 minutos para todos los centros de Atención Primaria de la organización sanitaria integrada, por cada habitante del municipio donde se encuentra ubicado le corresponde una disminución del tiempo en 0,00008 minutos, de tal forma que la estimación es, por ejemplo, en el caso de Vitoria con una población de 242254 habitantes: 29,089 minutos + $((-0,00008) * 242.254$ habitantes $)=10,51$ minutos.

El coeficiente de determinación $\mathrm{R}^{2}$, que mide el porcentaje de explicación de la población respecto al tiempo de acceso, para el caso que nos ocupa, podemos decir que el número de habitantes de Vitoria determina ese tiempo de acceso en un 69,24\%. En el parámetro de distancia en kilómetros, el valor de la R de Pearson es -0,837 y la ecuación de la recta y =26,942 - 0,00010x. En este caso también se puede interpretar que partiendo de un valor inicial de 26,942 kilómetros para todos los centros de Atención Primaria de la organización sanitaria integrada, por cada habitante del municipio donde se encuentra ubicado le corresponde una disminución de la distancia en 0,0001 kilómetros, de tal forma que la estimación es, por ejemplo, en el caso de Vitoria con una población de 242254 habitantes: 26,942 kilómetros + ((-0,0001) * 242254 habitantes) = 2,72 kilómetros.

El coeficiente de determinación $\mathrm{R}^{2}$, que mide el porcentaje de explicación de la población respecto la distancia de acceso, en kilómetros, para el caso que nos ocupa, podemos decir que el número de habitantes de Vitoria determina la distancia de acceso en un $70,14 \%$. Otras con ajustes de correlación negativa, superiores a -0,50 son Donostialdea (-0,567 tiempo / -0,563 distancia) y Alto Deba (-0,529 tiempo / -0,601 distancia). El resto de organizaciones sanitarias integradas, en la mayoría de los casos la correlación también es negativa, al igual que en las Áreas Sanitarias restantes, pero el valor no es significativo en ninguna de ellas.

La discusión del análisis de accesibilidad poblacional, en el País Vasco, al igual que sus tres Territorios Históricos, permite evidenciar que la mayor parte de la población, aproximadamente un $90 \%$, se encuentra entre una accesibilidad "normal" y "óptima", esto es, entre 0 y 20 minutos hasta su hospital de referencia y un porcentaje similar de población se encuentra a una distancia valorada entre normal y óptima, es decir desde 0 hasta 20 kilómetros:

- País Vasco: Tiempo: 1952815 hab. (89,75 \%) / Distancia: 1942701 hab. (89,29%).

- Araba/Álava: Tiempo: 274768 hab. (84,91 \%) / Distancia: 281121 hab. (86,88 \%).

- Bizkaia: Tiempo: 1017077 hab. (89,26 \%) / Distancia: 1011406 hab. (88,76 \%).

- Gipuzkoa: Tiempo: 660970 hab. (92,73 \%) / Distancia: 650174 hab. (91,21\%).

Visto lo cual, resulta necesario, en la medida de lo posible, actuar sobre ese aproximadamente $10 \%$ de la población que se encuentra más desfavorecida. Se debe empezar por aquellas organizaciones sanitarias integradas, cuyos municipios se encuentran en una situación de accesibilidad y distancia calificada como "inaceptable", superiores a 30 minutos y/o 30 kilómetros de distancia, por lo que las posibles áreas de mejora se explicitarán, a continuación, en el mismo apartado que las conclusiones a este artículo.

Para finalizar la discusión y desde un punto de vista comparativo con otros trabajos precedentes, de ámbito nacional, cuyo objetivo sea más equiparable al presente, podemos decir que respecto al estudio de accesibilidad de la población rural a los servicios básicos de salud, de la provincia de Teruel, Escalona y Díez, (2003), cuyos intervalos temporales son menos exigentes y se refieren a los recursos de Atención Primaria, los resultados del País Vasco son mejores, incluso siendo más estrictos y tratándose de recursos de Atención Especializa. Es cierto, que las características de la provincia de Teruel, por su escasa densidad de población y extensión territorial, son difícilmente comparables al País Vasco y ello se traduce en los resultados.

También referido al sistema sanitario español y utilizando los mismos intervalos que el artículo sobre la provincia de Teruel, la Tesis Doctoral de Rodríguez, (2014), "Accesibilidad geográfica de la población a la red de hospitales públicos de Andalucía: aportaciones desde el análisis de redes" analiza el tiempo de viaje en el ámbito municipal para el primer, segundo y tercer nivel de especialización de la red de hospitales públicos de Andalucía. Existe coincidencia con este trabajo, en uno y otro se refiere a los hospitales públicos, aunque los intervalos al igual que el anterior, son menos exigentes en Andalucía. También se evidencia, a la vista de 
los resultados, que los del País Vasco son algo mejores ya que, aunque hasta el intervalo de 15 minutos son parecidos en uno y otro caso, a partir de ahí, los tiempos de desplazamiento en Andalucía se incrementan notablemente. La razón, se debe también, a la disparidad entre la densidad de población y extensión entre las dos Comunidades Autónomas y tal como se menciona en la propia tesis, hay municipios andaluces de las áreas de Andévalo, Sierra de Cazorla, Segura y las Villas y Alpujarras que la conexión de carreteras es de tercer orden y se encuentran alejadas del hospital de referencia.

\section{CONCLUSIONES}

Este estudio ha permitido evaluar la distribución territorial de los hospitales del País Vasco (Osakidetza), estableciendo un nivel de cobertura territorial en el $90 \%$ de los municipios.

El interés del estudio, además del mero conocimiento en la distribución óptima de los recursos sanitarios en el País Vasco, estriba en hacer converger la ciencia geográfica con la distribución de dichos recursos y junto a ellos, la sostenibilidad económica fruto de una asignación geográfica eficiente de los hospitales.

Los restantes municipios (10 \% del estudio), que precisan de una actuación más decidida para mejorar sus parámetros son, de acuerdo con los resultados expuestos y los que se evidencian, a simple vista, (ver mapas 4 y 5) los correspondientes a los municipios o comarcas de las siguientes provincias u organizaciones sanitarias integradas:

En Ezkerraldea-Enkarterri-Cruces los municipios más próximos a la Comunidad Autónoma de Cantabria, en el oeste de Bizkaia; en Barrualde-Galdakao, la Comarca de Lea-Artibai, en la zona nororiental de Bizkaia y los municipios del norte de Araba/Álava, de la Comarca de Ayala o Cantabrico-Alavesa; en la organización sanitaria integrada Araba, los municipios con peor accesibilidad pertenecen a las comarcas de la Montaña Alavesa; en Goierri-Alto Urola, los municipios con peor accesibilidad pertenecen a la comarca de Gipuzkoa denominada Urola-Kosta, desde la zona costera central de Gipuzkoa, hacia el sur del territorio.

Para cada situación descrita puede haber una o varias soluciones o áreas de mejora que, si bien no subsanan totalmente el problema de accesibilidad, pudieran mejorarla de aplicarse, como las siguientes:

- Reorganización del mapa sanitario de las organizaciones sanitarias integradas. Aunque este estudio no profundiza sobre aspectos internos de estas organizaciones, por lo que sería temerario opinar sobre cuestiones de funcionamiento, capacidad, cobertura poblacional etc., se debería estudiar la posibilidad de reorganizar el mapa sanitario en algunas, para optimizar su accesibilidad. Por ejemplo, los municipios de Ondarroa, Berriatua y Markina, de Barrualde-Galdakao, no cubiertos por el Hospital de Gernika, mejorarían su accesibilidad si estuvieran adscritos a Debabarrena, acudiendo al Hospital Mendaro, igual que otros municipios vizcaínos como Mallabia y Ermua. Algunos municipios de Goierri-Alto Urola, como Azkoitia y Azpeitia, de Gipuzkoa, también mejorarían su accesibilidad adscribiéndose a Debabarrena y en el caso del municipio de Errezil (Gipuzkoa) a la OSI Tolosaldea.

- Establecimiento de Convenios de Colaboración con Comunidades Autónomas vecinas. Algunos municipios se encuentran más próximos a hospitales pertenecientes a otras Comunidades Autónomas que el correspondiente a su organización sanitaria integrada. Al mismo tiempo, el establecimiento de convenios de colaboración para la asistencia sanitaria supracomunitaria no es una figura extraña, ya que existen precedentes de esta forma de solidaridad. La organización sanitaria integrada Bilbao-Basurto, por ejemplo, es referente en determinadas prestaciones de carácter supraterritorial al Valle de Mena (Burgos), perteneciente a Castilla y León. Por tanto, es razonable pensar la reciprocidad entre comunidades puede ser extensiva como en el caso de la Rioja donde existen acuerdos de colaboración en materia de asistencia sanitaria mutua. Por esta vía, de llegarse a convenios de colaboración con otras comunidades vecinas, en alguno de los municipios más próximos a Cantabria como el Valle de Carranza, Lanestosa y Trucios etc., dependientes de Ezkerraldea-Enkarterri-Cruces, su accesibilidad mejoraría adscribiendo la asistencia sanitaria especializada de su población al Hospital Comarcal de Laredo (Cantabria). En la organización sanitaria integrada Araba, para la comarca de la Montaña Alavesa, algunos municipios se encuentran más próximos, por ejemplo, al Hospital 
Santiago Apóstol de Miranda de Ebro (Burgos) en Castilla y León y en el caso de la comarca de los Valles Alaveses, ocurre otro tanto con el Hospital García Orcoyen de Estella (Navarra).

- Mejorar las infraestructuras de comunicaciones por carretera y el transporte público interurbano. Una forma de acercar la accesibilidad a los dispositivos sanitarios puede conseguirse actuando sobre las infraestructuras de comunicaciones: autopistas, carreteras, transporte público etc. No obstante, hay que recordar que esta responsabilidad no corresponde a la Administración Sanitaria, puesto que en el País Vasco la competencia de la gestión de las carreteras y el transporte público interurbano depende, generalmente, de las Diputaciones Forales de cada uno de los Territorio Históricos.

Por último, cabe señalar que las anteriores áreas de mejora, basadas en los resultados y discusión del trabajo, no son una lista cerrada y quizás sea posible adoptar otras medidas distintas para conseguir un resultado similar de accesibilidad y equidad en el acceso a los recursos sanitarios. En cualquier caso, las administraciones públicas, para prestar sus servicios deben adaptarse a las realidades geográficas y sociales de sus territorios (tamaño, orografía, condiciones meteorológicas, población, etc.) y las soluciones que son válidas para unos, pueden no serlo tanto para otros. A modo de ejemplo, otras soluciones alternativas que pueden implementarse, son el transporte sanitario colectivo para habitantes de las comarcas rurales o dotarse de una red de helipuertos sanitarios de emergencia en las zonas más inaccesibles y/o alejadas de los hospitales, este último se viene aplicando en Andalucía desde hace unos años. (Comisión Europea, s.f.).

\section{Declaración responsable y conflicto de intereses}

Las/os autoras/es declaran que no existe ningún conflicto de interés con relación a la publicación de este artículo. Las tareas se han distribuido de la siguiente manera: Las/os dos autoras/es han participado en la revisión bibliográfica, el diseño de la metodología y la redacción del artículo. La realización del análisis gráfico y cuantitativo ha estado a cargo de Javier Cano Fernández.

\section{REFERENCIAS}

Almeida, G. (2020, 14 de febrero). Monitoreo y evaluación de la equidad en salud y servicios de salud. Organización Panamericana de Salud. https://www.paho.org/hq/index.php?option=com_docman\&view=list\&format=html\&layout=default\&slug=analisis-sistemas-servicios-salud-1990\&Itemid=270\&lang=es

Barbieri, N. C., Puente, C. \& Tarragona, S. (2002). La equidad en el gasto público en salud. Serie de Estudios en Finanzas Públicas, 9, 5. http://sedici.unlp.edu.ar/handle/10915/3486

Basoa-Rivas, G. \& Otero-Puyme, A. (1994). Accesibilidad geográfica a los centros de salud y planteamiento urbanístico en Fuenlabrada (Madrid). Revista de sanidad e higiene pública, 68 (4), 503-511.

Betancourth-Espinales, S.G. (2011). Equidad espacial en atención primaria en salud, Municipio León Nicaragua [Tesis de Grado, Universidad San Francisco de Quito]. Repositorio Institucional USFQ. http://repositorio.usfq.edu.ec/handle/23000/1644

Buzai, G.D. (2011). Modelos de localización-asignación aplicados a servicios públicos urbanos: análisis espacial de Centros de Atención Primaria de Salud (CAPS) en la ciudad de Luján, Argentina. Cuadernos de geografía: Revista Colombiana de Geografía, 20 (2). https://doi.org/10.15446/rcdg.v20n2.27184

Camarero, L. \& Oliva, J. (2019). Thinking in rural gap: mobility and social inequalities. Palgrave Commun 5, 95. https://doi. org/10.1057/s41599-019-0306-x

Comisión Europea (2020, 1 de diciembre). Las instalaciones sanitarias andaluzas mejoran su rendimiento. https://ec.europa.eu/regional_policy/es/projects/spain/healthcare-facilities-in-andalusia-achieve-a-higher-performance

Escalona-Orcao, A. I. \& Díez-Cornago, C. (2003). Accesibilidad geográfica de la población rural a los servicios básicos de salud: estudio en la provincia de Teruel. Ager. Revista de Estudios sobre Despoblación y Desarrollo Rural, 3, 129. http:// ruralager.org/wp-content/uploads/Ager-03_4.pdf 
Gang, C., Xiankai, Z., Lian, D., Xiaoping, L., Huichao, S., Tao, J. \& Yuli, L. (2016). Spatial difference analysis for accessibility to high level hospitals based on travel time in Shenzhen, China. Habitat International, 53, 485-494. https://doi.org/10.1016/j.habitatint.2015.12.023

Gobierno Vasco (2019, 20 de diciembre). Geoeuskadi, Infraestructura de Datos Espaciales (IDE) de Euskadi. http://www.geo. euskadi.eus

Instituto Vasco de Estadística (Eustat) (2020, 15 de enero). Población 2017. http://www.eustat.eus/estadisticas/tema_159/ opt_0/tipo_1/ti_Poblacion/temas.html

Infosalus (2013). Sanidad cumple con distancia máxima de 30 minutos a todos los centros de Salud tras cotejar datos con Fomento. http://www.infosalus.com/asistencia/noticia-cmancha-sanidad-cumple-distancia-maxima-30-minutos-todos-centros-salud-cotejar-datos-fomento-20130118135834.html

López-Lara, E. \& Garrido-Cumbrera, M. (2003). Análisis de la accesibilidad hospitalaria por carretera en Andalucía mediante Sistemas de Información Geográfica. En E. López-Lara (coord..), Servicios y transportes en el desarrollo territorial de España (405-416). https://idus.us.es/handle/11441/64401

López-Lara, E., Garrido-Cumbrera, M. \& Díaz-Cuevas, M. P. (2012). Improving territorial accessibility of mental health services: The case of Spain. Eur. J. Psychiat, 26 (4), 227-235. https://doi.org/10.4321/S0213-61632012000400002

Loyola, E., Castillo-Salgado, C., Nájera-Aguilar, P., Vidaurre, M., Mújica, O. J. \& Martínez-Piedra, R. (2002). Los sistemas de información geográfica como herramienta para monitorear las desigualdades de salud. Revista Panamericana de Salud Pública, 12 (6), 415-428. https://scielosp.org/pdf/rpsp/v12n6/a09v12n6.pdf

Osakidetza (2019, 12 de diciembre). Recursos del Sistema Sanitario Público Vasco. https://www.etxebide.euskadi.eus/ x39-contgen/es/contenidos/informacion/recursos_sistema_sanitario/es_sanidad/index.shtml

Palomar-Rodríguez, A., Mas-Castillo, A., Parra-Hidalgo, P. \& Rodríguez-Martínez, M. (1996). Accesibilidad a los dispositivos sanitarios de la Región de Murcia. Atención Primaria,17(8) http://www.elsevier.es/es-revista-atencion-primaria-27-articulo-accesibilidad-los-dispositivos-sanitarios-region-14259

Pan H., Zhen L. \& Jing L. (2019). Equity and Efficiency in Spatial Distribution of Basic Public Health Facilities: A Case Study from Nanjing Metropolitan Area. Urban Policy and Research, 37(2), 243-266. https://doi.org/10.1080/08111146.201 8.1523055

Ramírez M. L. \& Bosque-Sendra, J. (2001). Localización de hospitales: analogías y diferencias del uso del modelo p-mediano en SIG raster y vectorial. Anales de Geografía de la Universidad Complutense, 21, 53-79.

Reyes-Blanco, O. \& Franklin-Sam, O. R. (2016). Teoría del bienestar y el óptimo de Pareto como problemas microeconómicos. La Calera, 14 (22), 50-56. https://doi.org/10.5377/calera.v14i22.2657

Rodríguez-Díaz, V. (2014). Accesibilidad geográfica de la población a la red de hospitales públicos de Andalucía: aportaciones desde el análisis de redes [Tesis Doctoral, Universidad Pablo Olavide]. Repositorio Institucional UPO http://hdl. handle.net/10433/1534

Rodríguez- Díaz, V. (2011). Medición de la accesibilidad geográfica de la población a los hospitales de alta resolución Andalucía. Revista Internacional de Ciencia y Tecnología de la Información Geográfica. http://www.geofocus.org/index. php/geofocus/article/view/220

Smith, D. M. (1980). Geografía Humana. Serie elementos de geografía. Oikus-Tau.

Tobar, F., Montiel, L., Gaya, R. \& Martínez, E. (2001). Modelos equitativos de distribución de recursos sanitarios. En Resultado parcial proyecto investigación: Propuestas para un modelo de federalismo sanitario en Argentina. Buenos Aires. (1), 1.

Unidad Docente Medicina de Familia y Comunitaria de Mérida (2019, 14 de mayo). Descripción física del Área de Salud de Mérida. http://udfamiliamerida.es/index.php/area-sanitaria

Whitehead, M. (1991). Los conceptos y principios de la equidad en la salud. OPS/OMS. Programa de Desarrollo de Políticas de Salud. Serie Traducciones. (1), 7. http://onis.salud.gob.mx/site4/somos/docs/taller_analisis_datos_bibliografia_27.pdf

Whitehead, J., L. Pearson, A., Lawrenson, R. \& Atatoa-Carr, P. (2019). How can the spatial equity of health services be defined and measured? A systematic review of spatial equity definitions and methods. Journal of health services research \& policy, 24(4), 270-278. https://doi.org/10.1177/1355819619837292 


\section{ANEXOS}

Tabla 8. Accesibilidad población provincial, por intervalos.

\begin{tabular}{|c|c|c|c|c|c|c|c|}
\hline \multicolumn{8}{|c|}{ ARABA / ÁLAVA } \\
\hline TIEMPO & Municipios & Población & $\%$ & DISTANCIA & Municipios & Población & $\%$ \\
\hline Entre 0 y 5 minutos & 0 & 0.000 & $0,00 \%$ & Entre 0 y 5 km. & 4 & 243.084 & $75,12 \%$ \\
\hline De 6 a 10 minutos & 5 & 244.633 & $75,60 \%$ & Más de 5 y hasta 10 km. & 7 & 6.016 & $1,86 \%$ \\
\hline De 11 a 15 minutos & 5 & 2.795 & $0,86 \%$ & Más de 10 y hasta 15 km. & 4 & 6.625 & $2,05 \%$ \\
\hline De 16 a 20 minutos & 8 & 27.340 & $8,45 \%$ & Más de 15 y hasta 20 km. & 7 & 25.396 & $7,85 \%$ \\
\hline De 21 a 25 minutos & 8 & 14.127 & $4,37 \%$ & Más de 20 y hasta 25 km. & 4 & 5.430 & $1,68 \%$ \\
\hline De 26 a 30 minutos & 12 & 25.065 & $7,75 \%$ & Más de 25 y hasta 30 km. & 10 & 14.641 & $4,52 \%$ \\
\hline Más de 30 minutos & 13 & 9.632 & $2,98 \%$ & Más de 30 km. & 15 & 22.400 & $6,92 \%$ \\
\hline TOTAL & 51 & 323.592 & $100,00 \%$ & TOTAL & 51 & 323.592 & $100,00 \%$ \\
\hline
\end{tabular}

\begin{tabular}{|c|c|c|c|c|c|c|c|}
\hline \multicolumn{8}{|c|}{ ARABA / ÁLAVA } \\
\hline TIEMPO & Municipios & Población & $\%$ & DISTANCIA & Municipios & Población & $\%$ \\
\hline Entre 0 y 5 minutos & 7 & 23.947 & $2,10 \%$ & Entre 0 y $5 \mathrm{~km}$. & 16 & 545.825 & $47,90 \%$ \\
\hline De 6 a 10 minutos & 14 & 189.208 & $16,61 \%$ & Más de 5 y hasta 10 km. & 20 & 209.681 & $18,40 \%$ \\
\hline De 11 a 15 minutos & 27 & 631.628 & $55,43 \%$ & Más de 10 y hasta 15 km. & 23 & 195.270 & $17,14 \%$ \\
\hline De 16 a 20 minutos & 22 & 172.294 & $15,12 \%$ & Más de 15 y hasta 20 km. & 19 & 60.630 & $5,32 \%$ \\
\hline De 21 a 25 minutos & 18 & 63.385 & $5,56 \%$ & Más de 20 y hasta 25 km. & 17 & 78.254 & $6,87 \%$ \\
\hline De 26 a 30 minutos & 12 & 33.267 & $2,92 \%$ & Más de 25 y hasta 30 km. & 7 & 11.533 & $1,01 \%$ \\
\hline Más de 30 minutos & 12 & 25.697 & $2,26 \%$ & Más de 30 km. & 10 & 38.233 & $3,36 \%$ \\
\hline TOTAL & 112 & 1.139 .426 & $100,00 \%$ & TOTAL & 112 & 1.139 .426 & $100,00 \%$ \\
\hline
\end{tabular}

\begin{tabular}{|c|c|c|c|c|c|c|c|}
\hline \multicolumn{8}{|c|}{ GIPUZKOA } \\
\hline TIEMPO & Municipios & Población & $\%$ & DISTANCIA & Municipios & Población & $\%$ \\
\hline Entre 0 y 5 minutos & 3 & 28.407 & $3,99 \%$ & Entre 0 y $5 \mathrm{~km}$. & 14 & 170.922 & $23,98 \%$ \\
\hline De 6 a 10 minutos & 19 & 366.105 & $51,36 \%$ & Más de 5 y hasta 10 km. & 24 & 318.340 & $44,66 \%$ \\
\hline De 11 a 15 minutos & 26 & 137.520 & $19,29 \%$ & Más de 10 y hasta 15 km. & 26 & 122.507 & $17,19 \%$ \\
\hline De 16 a 20 minutos & 26 & 128.938 & $18,09 \%$ & Más de 15 y hasta 20 km. & 12 & 38.405 & $5,39 \%$ \\
\hline De 21 a 25 minutos & 6 & 19.402 & $2,72 \%$ & Más de 20 y hasta 25 km. & 7 & 45.641 & $6,40 \%$ \\
\hline De 26 a 30 minutos & 6 & 16.984 & $2,38 \%$ & Más de 25 y hasta 30 km. & 1 & 2.025 & $0,28 \%$ \\
\hline Más de 30 minutos & 2 & 15.445 & $2,17 \%$ & Más de 30 km. & 4 & 14.961 & $2,10 \%$ \\
\hline TOTAL & 88 & 712.801 & $100,00 \%$ & TOTAL & 88 & 712.801 & $100,00 \%$ \\
\hline
\end{tabular}

Fuente: Elaboración propia, a partir de datos de Osakidetza, Eustat y Google Earth. 
Tabla 9. Accesibilidad población organizaciones sanitarias integradas Araba/Álava, por intervalos

\begin{tabular}{|c|c|c|c|c|c|c|c|}
\hline \multicolumn{8}{|c|}{ ORGANIZACIÓN SANITARIA INTEGRADA ÁRABA } \\
\hline TIEMPO & Municipios & Población & $\%$ & DISTANCIA & Municipios & Población & $\%$ \\
\hline Entre 0 y 5 minutos & 0 & 0 & $0,00 \%$ & Entre 0 y $5 \mathrm{~km}$. & 1 & 242.254 & $87,33 \%$ \\
\hline De 6 a 10 minutos & 1 & 242.254 & $87,33 \%$ & Más de 5 y hasta 10 km. & 1 & 1.028 & $0,37 \%$ \\
\hline De 11 a 15 minutos & 0 & 0 & $0,00 \%$ & Más de 10 y hasta 15 km. & 3 & 5.751 & $2,07 \%$ \\
\hline De 16 a 20 minutos & 3 & 5.050 & $1,82 \%$ & Más de 15 y hasta 20 km. & 3 & 4.624 & $1,67 \%$ \\
\hline De 21 a 25 minutos & 6 & 7.817 & $2,82 \%$ & Más de 20 y hasta 25 km. & 5 & 3.517 & $1,27 \%$ \\
\hline De 26 a 30 minutos & 10 & 14.547 & $5,24 \%$ & Más de 25 y hasta 30 km. & 7 & 11.223 & $4,05 \%$ \\
\hline Más de 30 minutos & 12 & 7.734 & $2,79 \%$ & Más de 30 km. & 12 & 9.005 & $3,25 \%$ \\
\hline TOTAL & 32 & 277.402 & $100,00 \%$ & TOTAL & 32 & 277.402 & $100,00 \%$ \\
\hline
\end{tabular}

\begin{tabular}{|c|c|c|c|c|c|c|c|}
\hline \multicolumn{8}{|c|}{ ORGANIZACIÓN SANITARIA INTEGRADA ERRIOXA ARABAKO-RIOJA ALAVESA } \\
\hline TIEMPO & Municipios & Población & $\%$ & DISTANCIA & Municipios & Población & $\%$ \\
\hline Entre 0 y 5 minutos & 0 & 0 & $0,00 \%$ & Entre 0 y 5 km. & 3 & 830 & $7,21 \%$ \\
\hline De 6 a 10 minutos & 4 & 2.379 & $20,67 \%$ & Más de 5 y hasta 10 km. & 5 & 3.470 & $30,15 \%$ \\
\hline De 11 a 15 minutos & 5 & 2.795 & $24,29 \%$ & Más de 10 y hasta $15 \mathrm{~km}$. & 1 & 874 & $7,59 \%$ \\
\hline De 16 a 20 minutos & 3 & 2.373 & $20,62 \%$ & Más de 15 y hasta 20 km. & 3 & 2.373 & $20,62 \%$ \\
\hline De 21 a 25 minutos & 1 & 3.427 & $29,78 \%$ & Más de 20 y hasta 25 km. & 1 & 3.427 & $29,78 \%$ \\
\hline De 26 a 30 minutos & 2 & 535 & $4,65 \%$ & Más de 25 y hasta 30 km. & 2 & 535 & $4,65 \%$ \\
\hline Más de 30 minutos & 0 & 0 & $0,00 \%$ & Más de 30 km. & 0 & 0 & $0,00 \%$ \\
\hline TOTAL & 15 & 11.509 & $100,00 \%$ & TOTAL & 15 & 11.509 & $100,00 \%$ \\
\hline
\end{tabular}

Fuente: Elaboración propia, a partir de datos de Osakidetza, Eustat y Google Earth. 
Tabla 10. Accesibilidad población organizaciones sanitarias integradas Bizkaia, por intervalos.

\begin{tabular}{|c|c|c|c|c|c|c|c|}
\hline \multicolumn{8}{|c|}{ ORGANIZACIÓN SANITARIA INTEGRADA BARAKALDO-SESTAO } \\
\hline TIEMPO & Municipios & Población & $\%$ & DISTANCIA & Municipios & Población & $\%$ \\
\hline Entre 0 y 5 minutos & 0 & 0 & $0,00 \%$ & Entre 0 y $5 \mathrm{~km}$. & 2 & 125.607 & $100,00 \%$ \\
\hline De 6 a 10 minutos & 2 & 125.607 & $100,00 \%$ & Más de 5 y hasta 10 km. & 0 & 0 & $0,00 \%$ \\
\hline De 11 a 15 minutos & 0 & 0 & $0,00 \%$ & Más de 10 y hasta $15 \mathrm{~km}$. & 0 & 0 & $0,00 \%$ \\
\hline De 16 a 20 minutos & 0 & 0 & $0,00 \%$ & Más de 15 y hasta 20 km. & 0 & 0 & $0,00 \%$ \\
\hline De 21 a 25 minutos & 0 & 0 & $0,00 \%$ & Más de 20 y hasta 25 km. & 0 & 0 & $0,00 \%$ \\
\hline De 26 a 30 minutos & 0 & 0 & $0,00 \%$ & Más de 25 y hasta 30 km. & 0 & 0 & $0,00 \%$ \\
\hline Más de 30 minutos & 0 & 0 & $0,00 \%$ & Más de 30 km. & 0 & 0 & $0,00 \%$ \\
\hline TOTAL & 2 & 125.607 & $100,00 \%$ & TOTAL & 2 & 125.607 & $100,00 \%$ \\
\hline
\end{tabular}

\begin{tabular}{|c|c|c|c|c|c|c|c|}
\hline \multicolumn{8}{|c|}{ ORGANIZACIÓN SANITARIA INTEGRADA BARRUALDE-GALDAKAO } \\
\hline TIEMPO & Municipios & Población & $\%$ & DISTANCIA & Municipios & Población & $\%$ \\
\hline Entre 0 y 5 minutos & 6 & 19.852 & $6,46 \%$ & Entre 0 y 5 km. & 10 & 53.934 & $17,56 \%$ \\
\hline De 6 a 10 minutos & 8 & 39.817 & $12,97 \%$ & Más de 5 y hasta 10 km. & 10 & 58.998 & $19,21 \%$ \\
\hline De 11 a 15 minutos & 15 & 100.702 & $32,79 \%$ & Más de 10 y hasta 15 km. & 13 & 46.727 & $15,22 \%$ \\
\hline De 16 a 20 minutos & 15 & 65.840 & $21,44 \%$ & Más de 15 y hasta 20 km. & 11 & 44.009 & $14,33 \%$ \\
\hline De 21 a 25 minutos & 8 & 30.258 & $9,85 \%$ & Más de 20 y hasta 25 km. & 9 & 50.099 & $16,31 \%$ \\
\hline De 26 a 30 minutos & 8 & 28.135 & $9,16 \%$ & Más de 25 y hasta 30 km. & 7 & 13.589 & $4,42 \%$ \\
\hline Más de 30 minutos & 8 & 22.506 & $7,33 \%$ & Más de 30 km. & 8 & 39.754 & $12,94 \%$ \\
\hline TOTAL & 68 & 307.110 & $100,00 \%$ & TOTAL & 68 & 307.110 & $100,00 \%$ \\
\hline
\end{tabular}

\begin{tabular}{|c|c|c|c|c|c|c|c|}
\hline \multicolumn{8}{|c|}{ ORGANIZACIÓN SANITARIA INTEGRADA BILBAO-BASURTO } \\
\hline TIEMPO & Municipios & Población & $\%$ & DISTANCIA & Municipios & Población & $\%$ \\
\hline Entre 0 y 5 minutos & 0 & 0 & $0,00 \%$ & Entre 0 y 5 km. & 1 & 342.397 & $99,18 \%$ \\
\hline De 6 a 10 minutos & 1 & 2.837 & $0,82 \%$ & Más de 5 y hasta 10 km. & 1 & 2.837 & $0,82 \%$ \\
\hline De 11 a 15 minutos & 1 & 342.397 & $99,18 \%$ & Más de 10 y hasta 15 km. & 0 & 0 & $0,00 \%$ \\
\hline De 16 a 20 minutos & 0 & 0 & $0,00 \%$ & Más de 15 y hasta 20 km. & 0 & 0 & $0,00 \%$ \\
\hline De 21 a 25 minutos & 0 & 0 & $0,00 \%$ & Más de 20 y hasta 25 km. & 0 & 0 & $0,00 \%$ \\
\hline De 26 a 30 minutos & 0 & 0 & $0,00 \%$ & Más de 25 y hasta 30 km. & 0 & 0 & $0,00 \%$ \\
\hline Más de 30 minutos & 0 & 0 & $0,00 \%$ & Más de 30 km. & 0 & 0 & $0,00 \%$ \\
\hline TOTAL & 2 & 345.234 & $100,00 \%$ & TOTAL & 2 & 345.234 & $100,00 \%$ \\
\hline
\end{tabular}




\begin{tabular}{|c|c|c|c|c|c|c|c|}
\hline \multicolumn{8}{|c|}{ ORGANIZACIÓN SANITARIA INTEGRADA EZKERRALDEA-ENKARTERRI-CRUCES } \\
\hline TIEMPO & Municipios & Población & $\%$ & DISTANCIA & Municipios & Población & $\%$ \\
\hline Entre 0 y 5 minutos & 0 & 0 & $0,00 \%$ & Entre 0 y 5 km. & 0 & 0 & $0,00 \%$ \\
\hline De 6 a 10 minutos & 0 & 0 & $0,00 \%$ & Más de 5 y hasta 10 km. & 2 & 54.682 & $33,59 \%$ \\
\hline De 11 a 15 minutos & 4 & 65.767 & $40,40 \%$ & Más de 10 y hasta $15 \mathrm{~km}$. & 4 & 68.679 & $42,19 \%$ \\
\hline De 16 a 20 minutos & 3 & 64.132 & $39,40 \%$ & Más de 15 y hasta 20 km. & 2 & 8.251 & $5,07 \%$ \\
\hline De 21 a 25 minutos & 3 & 17.639 & $10,84 \%$ & Más de 20 y hasta 25 km. & 3 & 18.474 & $11,35 \%$ \\
\hline De 26 a 30 minutos & 2 & 10.160 & $6,24 \%$ & Más de 25 y hasta 30 km. & 1 & 827 & $0,51 \%$ \\
\hline Más de 30 minutos & 5 & 5.089 & $3,13 \%$ & Más de 30 km. & 5 & 11.874 & $7,29 \%$ \\
\hline TOTAL & 17 & 162.787 & $100,00 \%$ & TOTAL & 17 & 162.787 & $100,00 \%$ \\
\hline
\end{tabular}

\begin{tabular}{|c|c|c|c|c|c|c|c|}
\hline \multicolumn{8}{|c|}{ ORGANIZACIÓN SANITARIA INTEGRADA URIBE } \\
\hline TIEMPO & Municipios & Población & $\%$ & DISTANCIA & Municipios & Población & $\%$ \\
\hline Entre 0 y 5 minutos & 1 & 4.095 & $1,91 \%$ & Entre 0 y 5 km. & 3 & 23.887 & $11,13 \%$ \\
\hline De 6 a 10 minutos & 3 & 20.947 & $9,76 \%$ & Más de 5 y hasta 10 km. & 7 & 93.164 & $43,41 \%$ \\
\hline De 11 a 15 minutos & 7 & 122.762 & $57,21 \%$ & Más de 10 y hasta 15 km. & 6 & 79.864 & $37,22 \%$ \\
\hline De 16 a 20 minutos & 4 & 44.647 & $20,80 \%$ & Más de 15 y hasta 20 km. & 5 & 9.516 & $4,43 \%$ \\
\hline De 21 a 25 minutos & 7 & 17.192 & $8,01 \%$ & Más de 20 y hasta 25 km. & 3 & 8.167 & $3,81 \%$ \\
\hline De 26 a 30 minutos & 2 & 4.955 & $2,31 \%$ & Más de 25 y hasta 30 km. & 0 & 0 & $0,00 \%$ \\
\hline Más de 30 minutos & 0 & 0 & $0,00 \%$ & Más de 30 km. & 0 & 0 & $0,00 \%$ \\
\hline TOTAL & 24 & 214.598 & $100,00 \%$ & TOTAL & 24 & 214.598 & $100,00 \%$ \\
\hline
\end{tabular}

Fuente: Elaboración propia, a partir de datos de Osakidetza, Eustat y Google Earth. 
Tabla 11. Accesibilidad población organizaciones sanitarias integradas Gipuzkoa, por intervalos.

\begin{tabular}{|c|c|c|c|c|c|c|c|}
\hline \multicolumn{8}{|c|}{ ORGANIZACIÓN SANITARIA INTEGRADA DONOSTIALDEA } \\
\hline TIEMPO & Municipios & Población & $\%$ & DISTANCIA & Municipios & Población & $\%$ \\
\hline Entre 0 y 5 minutos & 0 & 0 & $0,00 \%$ & Entre 0 y 5 km. & 2 & 25.638 & $7,15 \%$ \\
\hline De 6 a 10 minutos & 4 & 224.740 & $62,72 \%$ & Más de 5 y hasta 10 km. & 7 & 274.254 & $76,53 \%$ \\
\hline De 11 a 15 minutos & 5 & 68.851 & $19,21 \%$ & Más de 10 y hasta 15 km. & 1 & 10.290 & $2,87 \%$ \\
\hline De 16 a 20 minutos & 3 & 45.593 & $12,72 \%$ & Más de 15 y hasta 20 km. & 1 & 5.944 & $1,66 \%$ \\
\hline De 21 a 25 minutos & 1 & 3.825 & $1,07 \%$ & Más de 20 y hasta 25 km. & 2 & 25.859 & $7,22 \%$ \\
\hline De 26 a 30 minutos & 4 & 15.334 & $4,28 \%$ & Más de 25 y hasta 30 km. & 1 & 2.025 & $0,57 \%$ \\
\hline Más de 30 minutos & 0 & 0 & $0,00 \%$ & Más de 30 km. & 3 & 14.333 & $4,00 \%$ \\
\hline TOTAL & 17 & 358.343 & $100,00 \%$ & TOTAL & 17 & 358.343 & $100,00 \%$ \\
\hline
\end{tabular}

\begin{tabular}{|c|c|c|c|c|c|c|c|}
\hline \multicolumn{8}{|c|}{ ORGANIZACIÓN SANITARIA INTEGRADA GOIERRI-ALTO UROLA } \\
\hline TIEMPO & Municipios & Población & $\%$ & DISTANCIA & Municipios & Población & $\%$ \\
\hline Entre 0 y 5 minutos & 0 & 0 & $0,00 \%$ & Entre 0 y 5 km. & 2 & 16.678 & $17,51 \%$ \\
\hline De 6 a 10 minutos & 3 & 17.308 & $18,17 \%$ & Más de 5 y hasta 10 km. & 3 & 10.402 & $10,92 \%$ \\
\hline De 11 a 15 minutos & 5 & 25.152 & $26,41 \%$ & Más de 10 y hasta 15 km. & 5 & 15.890 & $16,68 \%$ \\
\hline De 16 a 20 minutos & 8 & 20.237 & $21,25 \%$ & Más de 15 y hasta 20 km. & 8 & 31.863 & $33,45 \%$ \\
\hline De 21 a 25 minutos & 4 & 15.451 & $16,22 \%$ & Más de 20 y hasta 25 km. & 5 & 19.782 & $20,77 \%$ \\
\hline De 26 a 30 minutos & 2 & 1.650 & $1,73 \%$ & Más de 25 y hasta 30 km. & 1 & 628 & $0,66 \%$ \\
\hline Más de 30 minutos & 2 & 15.445 & $16,22 \%$ & Más de 30 km. & 0 & 0 & $0,00 \%$ \\
\hline TOTAL & 24 & 95.243 & $100,00 \%$ & TOTAL & 24 & 95.243 & $100,00 \%$ \\
\hline
\end{tabular}

\begin{tabular}{|c|c|c|c|c|c|c|c|}
\hline \multicolumn{8}{|c|}{ ORGANIZACIÓN SANITARIA INTEGRADA BIDASOA } \\
\hline TIEMPO & Municipios & Población & $\%$ & DISTANCIA & Municipios & Población & $\%$ \\
\hline Entre 0 y 5 minutos & 0 & 0 & $0,00 \%$ & Entre 0 y 5 km. & 2 & 76.828 & $100,00 \%$ \\
\hline De 6 a 10 minutos & 2 & 76.828 & $100,00 \%$ & Más de 5 y hasta 10 km. & 0 & 0 & $0,00 \%$ \\
\hline De 11 a 15 minutos & 0 & 0 & $0,00 \%$ & Más de 10 y hasta 15 km. & 0 & 0 & $0,00 \%$ \\
\hline De 16 a 20 minutos & 0 & 0 & $0,00 \%$ & Más de 15 y hasta $20 \mathrm{~km}$. & 0 & 0 & $0,00 \%$ \\
\hline De 21 a 25 minutos & 0 & 0 & $0,00 \%$ & Más de 20 y hasta 25 km. & 0 & 0 & $0,00 \%$ \\
\hline De 26 a 30 minutos & 0 & 0 & $0,00 \%$ & Más de 25 y hasta 30 km. & 0 & 0 & $0,00 \%$ \\
\hline Más de 30 minutos & 0 & 0 & $0,00 \%$ & Más de 30 km. & 0 & 0 & $0,00 \%$ \\
\hline TOTAL & 2 & 76.828 & $100,00 \%$ & TOTAL & 2 & 76.828 & $100,00 \%$ \\
\hline
\end{tabular}




\begin{tabular}{|c|c|c|c|c|c|c|c|}
\hline \multicolumn{8}{|c|}{ ORGANIZACIÓN SANITARIA INTEGRADA DEBABARRENA (BAJO DEBA) } \\
\hline TIEMPO & Municipios & Población & $\%$ & DISTANCIA & Municipios & Población & $\%$ \\
\hline Entre 0 y 5 minutos & 1 & 2.135 & $2,93 \%$ & Entre 0 y $5 \mathrm{~km}$. & 1 & 2.135 & $2,93 \%$ \\
\hline De 6 a 10 minutos & 1 & 11.492 & $15,80 \%$ & Más de 5 y hasta 10 km. & 1 & 11.492 & $15,80 \%$ \\
\hline De 11 a 15 minutos & 0 & 0 & $0,00 \%$ & Más de 10 y hasta 15 km. & 4 & 41.871 & $57,55 \%$ \\
\hline De 16 a 20 minutos & 5 & 57.945 & $79,65 \%$ & Más de 15 y hasta 20 km. & 2 & 17.253 & $23,72 \%$ \\
\hline De 21 a 25 minutos & 1 & 1.179 & $1,62 \%$ & Más de 20 y hasta 25 km. & 0 & 0 & $0,00 \%$ \\
\hline De 26 a 30 minutos & 0 & & $0,00 \%$ & Más de 25 y hasta 30 km. & 0 & 0 & $0,00 \%$ \\
\hline Más de 30 minutos & 0 & & $0,00 \%$ & Más de 30 km. & 0 & 0 & $0,00 \%$ \\
\hline TOTAL & 8 & 72.751 & $100,00 \%$ & TOTAL & 8 & 72.751 & $100,00 \%$ \\
\hline
\end{tabular}

\begin{tabular}{|c|c|c|c|c|c|c|c|}
\hline \multicolumn{8}{|c|}{ ORGANIZACIÓN SANITARIA INTEGRADA ALTO DEBA } \\
\hline TIEMPO & Municipios & Población & $\%$ & DISTANCIA & Municipios & Población & $\%$ \\
\hline Entre 0 y 5 minutos & 1 & 22.047 & $34,13 \%$ & Entre 0 y 5 km. & 1 & 22.047 & $34,13 \%$ \\
\hline De 6 a 10 minutos & 1 & 4.100 & $6,35 \%$ & Más de 5 y hasta 10 km. & 3 & 12.591 & $19,49 \%$ \\
\hline De 11 a 15 minutos & 2 & 18.317 & $28,36 \%$ & Más de 10 y hasta 15 km. & 5 & 29.955 & $46,37 \%$ \\
\hline De 16 a 20 minutos & 5 & 20.129 & $31,16 \%$ & Más de 15 y hasta 20 km. & 0 & 0 & $0,00 \%$ \\
\hline De 21 a 25 minutos & 0 & 0 & $0,00 \%$ & Más de 20 y hasta 25 km. & 0 & 0 & $0,00 \%$ \\
\hline De 26 a 30 minutos & 0 & 0 & $0,00 \%$ & Más de 25 y hasta 30 km. & 0 & 0 & $0,00 \%$ \\
\hline Más de 30 minutos & 0 & 0 & $0,00 \%$ & Más de 30 km. & 0 & 0 & $0,00 \%$ \\
\hline TOTAL & 9 & 64.593 & $100,00 \%$ & TOTAL & 9 & 64.593 & $100,00 \%$ \\
\hline
\end{tabular}

\begin{tabular}{|c|c|c|c|c|c|c|c|}
\hline \multicolumn{8}{|c|}{ ORGANIZACIÓN SANITARIA INTEGRADA TOLOSALDEA } \\
\hline TIEMPO & Municipios & Población & $\%$ & DISTANCIA & Municipios & Población & $\%$ \\
\hline Entre 0 y 5 minutos & 1 & 4.225 & $6,62 \%$ & Entre 0 y $5 \mathrm{~km}$. & 6 & 27.596 & $43,24 \%$ \\
\hline De 6 a 10 minutos & 8 & 31.637 & $49,58 \%$ & Más de 5 y hasta 10 km. & 11 & 11.119 & $17,42 \%$ \\
\hline De 11 a 15 minutos & 14 & 25.200 & $39,49 \%$ & Más de 10 y hasta 15 km. & 11 & 24.501 & $38,39 \%$ \\
\hline De 16 a 20 minutos & 7 & 2.626 & $4,12 \%$ & Más de 15 y hasta 20 km. & 3 & 598 & $0,94 \%$ \\
\hline De 21 a 25 minutos & 1 & 126 & $0,20 \%$ & Más de 20 y hasta 25 km. & 0 & 0 & $0,00 \%$ \\
\hline De 26 a 30 minutos & 0 & 0 & $0,00 \%$ & Más de 25 y hasta 30 km. & 0 & 0 & $0,00 \%$ \\
\hline Más de 30 minutos & 0 & 0 & $0,00 \%$ & Más de 30 km. & 0 & 0 & $0,00 \%$ \\
\hline TOTAL & 31 & 63.814 & $100,00 \%$ & TOTAL & 31 & 63.814 & $100,00 \%$ \\
\hline
\end{tabular}

Fuente: Elaboración propia, a partir de datos de Osakidetza, Eustat y Google Earth. 